\title{
Coalescence and flow in ultra-relativistic heavy ion collisions
}

\author{
Rüdiger Scheibl and Ulrich Heinz \\ Institut für Theoretische Physik, Universität Regensburg, D-93040 Regensburg, Germany \\ (March 16, 1999 — published in Physical Review C 59 (1999), 1585-1602)
}

\begin{abstract}
Using a density matrix approach to describe the process of coalescence, we calculate the coalescence probabilities and invariant momentum spectra for deuterons and antideuterons. We evaluate our expressions with a hydrodynamically motivated parametrization for the source at freeze-out which implements rapid collective expansion of the collision zone formed in heavy ion collisions. We find that the coalescence process is governed by the same lengths of homogeneity which can be extracted from HBT interferometry. They appear in the absolute cluster yield via an effective volume factor as well as in a quantum mechanical correction factor which accounts for the internal structure of the deuteron cluster. Our analysis provides a new interpretation for the parameters in the popular phenomenological coalescence model and for the effective overlap volume in Hagedorn's model for cluster production in $p p$ collisions. Using source parameters extracted from a recent HBT analysis of two-pion correlations, we successfully describe deuteron and antideuteron production data from $\mathrm{Pb}+\mathrm{Pb}$ collisions at the CERN SPS as measured by the NA44 and NA52 collaborations. We also confirm the recent finding by Polleri et al. that the different measured slopes of nucleon and deuteron transverse mass spectra require a transverse density profile of the source which is closer to a box than to a Gaussian shape.
\end{abstract}

PACS numbers: 25.75.-q, 25.75.Ld, 25.75.Dw, 25.75.Gz

\section{INTRODUCTION}

Ultra-relativistic heavy ion collisions are used to study in the laboratory nuclear matter at extreme energy density and temperature. For such conditions lattice QCD predicts the transition from a hadron gas to a quarkgluon plasma. The only observables of the hot, central reaction zone (fireball) created in the heavy ion collision are the energies and the momenta of the produced particles. Most of these are hadrons which, due to their strong interactions, decouple only late from the collision fireball and therefore carry direct information only about this so-called "freeze-out stage". Direct signals from the earlier (presumably much hotter and denser) stages of the collision are carried by electromagnetic (photons, dileptons) and "hard" probes (jets, $J / \psi$ ) which are, however, more difficult to study due to their much smaller cross sections. Developing a clear and unique picture of the interesting early stages of ultra-relativistic heavy ion collisions will thus require the combination of such direct signatures with an extrapolation backwards in time of the information extracted from the spectra of the bulk of produced hadrons. For such a back-extrapolation to be useful, the analysis of the measured hadron spectra should permit a more or less complete reconstruction of both the geometry and the dynamics of the source at the point of freeze-out.

For this reason the last few years have seen a growing effort to understand the final state of heavy ion collisions quantitatively from the measured hadron spectra. In this project a crucial role is played by two-particle momentum correlations. Such correlations, whether due to final state interactions or quantum statistics, are sensi- tive to the phase-space distribution of the particles in the source at freeze-out and thus carry information not only on the momentum-space structure of the source, but also on its size and shape in coordinate space. In particular, they are sensitive to correlations between the momenta and positions of the particles at freeze-out such as those which are generated by the collective expansion of the hot and dense collision zone. This dynamical aspect is very important since, once identified and quantitatively analyzed, it carries valuable information about the early equilibration processes and the resulting build-up of thermodynamical (isotropic) pressure in the reaction zone, as well as on the time-integrated action of the equation of state of the quark-gluon and/or hadronic matter during the expansion stage.

The production of deuterons, antideuterons and larger nuclear clusters via coalescence of (anti-)nucleons at freeze-out can be viewed as a particular type of phasespace correlation among the particles in the final state. The similarity of the physics of coalescence and of other types of final state momentum correlations was stressed before by Mrówczyński [1] who pointed out that similar source information can be extracted from the analysis of both types of correlations. In the present paper we carry the analogy significantly farther by extending the investigation to systems with strong collective dynamics. We show in particular that the same "lengths of homogeneity in the source" [2] which can be extracted from two-particle Bose-Einstein correlations (Hanbury Brown - Twiss (HBT) interferometry [3]) determine the coalescence probability in the cluster formation process. This means that the effects of source expansion enter the "HBT radii" and the cluster yields in a similar way. For 
this reason nuclear cluster spectra provide an important and complementary check for the source reconstruction from single-particle spectra and two-particle momentum correlations of elementary hadrons.

As a point in case we demonstrate that we can successfully reproduce the measured cluster yields and spectra for deuterons, antideuterons, tritons and ${ }^{3} \mathrm{He}$ in $\mathrm{Pb}+\mathrm{Pb}$ collisions at the CERN SPS [4,6-8] with a source model whose parameters were determined [9, 10] from a combined analysis of pion spectra and two-pion correlations. We further show that the shape (inverse slope) of the measured deuteron transverse mass spectra [7,8, when compared with that of the proton spectra, yields additional constraints on the shape of the transverse source distribution in space which remove a specific ambiguity left open by the analysis of pion spectra and correlations.

Historically, cluster formation has been characterized in terms of the "invariant coalescence factor" $B_{A}$, defined through the invariant cluster momentum spectra via the equation

$$
E_{A} \frac{d N_{A}}{d^{3} P_{A}}=B_{A}\left(E_{\mathrm{p}} \frac{d N_{\mathrm{p}}}{d^{3} P_{\mathrm{p}}}\right)^{Z}\left(E_{\mathrm{n}} \frac{d N_{\mathrm{n}}}{d^{3} P_{\mathrm{n}}}\right)_{\mid P_{\mathrm{p}}=P_{\mathrm{n}}=P_{A} / A}^{N} .
$$

It relates the cluster spectrum to the invariant momentum spectra of the coalescing nucleons at the same velocity. The correct physical interpretation of $B_{A}$ has been a long-standing problem [1, 11, 26. The relative fragility of the nuclear clusters implies that only nucleons with small relative momenta contribute to cluster formation; this led early researchers [11 13 to an interpretation of $B_{A}$ in terms of a momentum space coalescence volume, parametrized in terms of a maximal relative momentum $p_{0}$ between the coalescing nucleons. This interpretation appeared to be confirmed by the approximate constancy of the $B_{A}$ values observed in heavy ion collisions at the BEVALAC with beam momenta up to about $1 \mathrm{GeV} /$ nucleon, independent of the beam energy and the size of the colliding nuclei. In the sudden approximation [14] and the thermodynamic [15] models, on the other hand, $B_{A} \sim V^{A-1}$ is inversely related to the fireball volume in coordinate space. In such a picture the decrease of the $B_{A}$ values with increasing beam energy, observed in nuclear collisions with large ions at beam energies above $1 \mathrm{GeV} /$ nucleon (for a recent compilation see 27]), can be easily understood in terms of collective expansion of the collision zone before break-up. Good reviews of these early approaches can be found in 16,19,26.

Later work began to stress more explicitly the phasespace aspects of the coalescence process, starting from a quantum mechanical approach based on the density matrix of the source or the equivalent Wigner function formalism 17, 18,20,22,23 and its classical phase-space analogues 21,24 . In this approach the size of the cluster itself enters as an additional dimensionful quantity into the calculation of $B_{A}$, and it allows to address the prob- lem of energy-momentum conservation in the coalescence process.

None of the existing model calculations accounts, however, properly for dynamical expansion of the source and the resulting correlations between the momenta and positions of the particles at freeze-out. These correlations can be included numerically by applying a density matrix or Wigner function based "coalescence afterburner" to the output of classical microscopic phase-space simulations like the Intranuclear Cascade [18], ARC [28], or RQMD [26,29,30]. While such numerical simulations may succeed or fail to reproduce the experimental data, in neither case they provide a clear conceptual understanding of the physics entering into the calculation of $B_{A}$. In this paper we present an analytical approach, based on an explicit source parametrization, which provides such an understanding. Like the static model calculations before, it is based on a quantum mechanical density matrix (Wigner function) approach. It thus allows for a proper treatment of energy-momentum conservation and to exhibit the dependence of $B_{A}$ on the phase-space structure of the source, i.e. on its longitudinal and transverse size and collective flow, and on the internal cluster structure.

The paper is organized as follows: In Sect. III we review shortly the classical thermal+flow model approach to cluster production in which clusters are viewed as pointlike elementary particles without internal structure which are produced by thermal emission from an expanding source. While poorly justified theoretically, this approach has enjoyed considerable phenomenological success. The results of Sect. II will serve as a benchmark for the discussion of the quantum mechanical density matrix approach presented in the following sections. We will discover strong formal similarities which simultaneously provide a theoretical justification for the phenomenological success of the thermal approach and yield an interpretation of the thermal model parameters for the cluster spectra in terms of the phase-space characteristics of the underlying nucleon source. This is described in Sects. III and [V] where we discuss the quantum mechanics of the coalescence process and calculate a quantum mechanical correction factor for the classical thermal cluster spectrum. We show that the latter is given by a very simple expression involving only the size of the cluster and the "homogeneity radii" of the source which can also be measured with HBT interferometry. In Sect. V we extend this discussion from two-nucleon clusters $(d$ and $\bar{d})$ to threenucleon clusters, $t$ and ${ }^{3} \mathrm{He}$. In Sect. VI we express the $B_{2}$ value in terms of of the HBT radii and show how cluster yields can be used for an analysis of the chemical composition of the fireball at the point of thermal nucleon freeze-out. This complements the chemical analysis of elementary hadron yields which determines the (usually much earlier) point of chemical freeze-out. A comparison of our results with heavy ion data is presented in Sect. VII, and our conclusions are presented in Sect. VIII.

We will use natural units $\hbar=c=k_{\mathrm{B}}=1$. We de- 
note by $m, m_{t}$, and $p_{t}$ the rest mass, transverse mass, and transverse momentum of a nucleon. $M, M_{t}$, and $P_{t}$ denote the respective variables of a cluster. For a cluster of $A$ nucleons we have in good approximation $M=A m$, $P=A p, P_{t}=A p_{t}$, and $M_{t}=A m_{t}$. We denote by $Y=y-y_{\mathrm{cm}}$ the longitudinal rapidity of a particle relative to c.m. frame of the fireball.

\section{THERMAL CLUSTER SPECTRA}

Clusters are no elementary hadrons. Their binding energies $(B=-2.25 \mathrm{MeV}$ for a deuteron) are small compared to typical collision energies in the fireball created in a heavy ion collision, and this makes them very fragile objects. While the spectra of elementary hadrons decoupling from a thermalized fireball along a freeze-out hypersurface $\Sigma_{\mathrm{f}}(R)$ with normal 4 -vector $d^{3} \sigma_{\mu}(R)$ can be described by the Cooper-Frye formula 31,

$$
E \frac{d^{3} N_{i}}{d^{3} P}=\frac{2 J+1}{(2 \pi)^{3}} \int_{\Sigma_{\mathrm{f}}} P \cdot d^{3} \sigma(R) f_{i}(R, P),
$$

where $f_{i}(R, P)$ is a local equilibrium distribution

$$
f_{i}(R, P)=\left[\exp \left(\frac{P \cdot u(R)-\mu_{i}(R)}{T(R)}\right) \pm 1\right]^{-1}
$$

with local temperature $T(R)$, local chemical potential $\mu_{i}(R)$, and local flow 4-velocity $u^{\mu}(R)$ (normalized to $u \cdot u=1$ ), a similar expression for nuclear clusters cannot be justified: clusters do not pre-exist as particles with thermalized momentum distributions in the collision fireball, but are only created by final state interactions among the nucleons during the freeze-out process.

The absence of preformed nuclear clusters in the collision zone of a relativistic heavy ion collision at AGS or SPS energies is easily seen: Nuclear fragmentation of the target and projectile nuclei can be excluded as the origin of cluster production if clusters made from antinucleons (e.g. $\bar{d}$ or ${ }^{3} \overline{\mathrm{H}}$ ) are selected. For clusters with positive baryon number near the midrapidity region it was estimated in 32] that the probability for a nuclear fragment to absorb the necessary longitudinal momentum transfer of several $\mathrm{GeV}$ to several $10 \mathrm{GeV}$ per nucleon without getting destroyed can be neglected. On the other hand, by applying Levinson's theorem 34 to the two-particle terms in the virial expansion of the grand canonical potential, it was shown in 35, 36. that thermal cluster production inside the fireball is exponentially suppressed when the binding energy of the cluster is much smaller than the fireball temperature.

In a very simple-minded approach to cluster formation at nucleon freeze-out one may postulate that a cluster of $A=Z+N$ nucleons with total momentum $P$ is emitted whenever $Z$ protons and $N$ neutrons of identical momenta $P / A$ happen to be at the same place $R$. This results in a generalized Cooper-Frye formula for clusters:

$$
\begin{aligned}
E \frac{d^{3} N_{A}}{d^{3} P}=\frac{2 J_{A}+1}{(2 \pi)^{3}} \int_{\Sigma_{\mathrm{f}}} P \cdot d^{3} \sigma(R) \\
\quad \times f_{\mathrm{p}}^{Z}(R, P / A) f_{\mathrm{n}}^{N}(R, P / A),
\end{aligned}
$$

where $f_{\mathrm{p}, \mathrm{n}}$ are given by Eq. (2.2). This formula agrees, up to a quantum mechanical correction factor which will be discussed below, with the Hagedorn model for cluster production in high energy $p p$ collisions 37. In spite of its naivity it has been quite successful [37,38]. It will be one of the main points of the present paper to explain why this is so. For this purpose we first analyze Eq. (2.3) for the specific source model which will later form the basis of our quantitative comparison with data.

\section{A. Analytical model for an expanding fireball}

There is mounting evidence from phenomenological analyses of existing data 39, 3, 9 as well as from microscopic simulations of the collision dynamics that during the early stages of a relativistic heavy ion collision the reaction zone approaches a state of local thermal equilibrium. The resulting thermal pressure causes a collective expansion of the system via hydrodynamic flow. It thus makes sense to parametrize the momentum distributions of the hadrons just before decoupling by Eq. (2.2), with the local flow velocity 4 -vector $u(R)$ describing the average particle velocity at point $R$ and $T(R)$ parametrizing the random ("thermal") local momentum fluctuations around their average value $p^{\mu}=m u^{\mu}(R)$. The local chemical potential $\mu_{i}(R)$ and the local fugacity $\lambda_{i}(R)=\exp \left[\mu_{i}(R) / T(R)\right]$ parametrize the local density of particle species $i$ at point $R$.

For heavy particles (nucleons and nuclear clusters) at chemical potentials $\mu_{i} \ll M_{i}$ (see below) Eq. (2.2) can be very well approximated by a local Boltzmann distribution. For simplicity we will assume freeze-out at constant temperature, $T_{\mathrm{f}}(R)=T=$ const. (General arguments based on the kinetics of the freeze-out process 41,42 show that this is generally a good approximation.) The fugacity $\lambda_{i}(R)$ is split into a constant, particle-specific term $\lambda_{i}=\exp \left(\mu_{i} / T\right)$, and a common density profile $\bar{H}(R)$ for all particle species. This implements the assumptions of local chemical equilibrium among the various particle species at freeze-out and simultaneous freeze-out of all particle species which seem reasonable for nucleons and nuclear clusters.

While in $\mathrm{Pb}+\mathrm{Pb}$ collisions in the initial state neutrons outnumber protons by a factor of 1.54 , the ratio $\mathrm{n} / \mathrm{p}$ or $\overline{\mathrm{n}} / \overline{\mathrm{p}}$ in the fireball at freeze-out is unknown. Due to particle production an unknown fraction of the initial isospin asymmetry in the nucleon sector may be transferred to other particle species. We introduce separate chemical potentials $\mu_{\mathrm{p}}$ and $\mu_{\mathrm{n}}$ for protons and neutrons and define the chemical potential of a cluster by $\mu_{A}=Z \mu_{\mathrm{p}}+N \mu_{\mathrm{n}}$. The values of $\mu_{\mathrm{p}}$ and $\mu_{\mathrm{n}}$ must be extracted from a fit to the data. 
We consider only very central (impact parameter $b \approx$ 0 ) collisions. The fireball is then azimutally symmetric with respect to the beam axis ("longitudinal" or $z$ axis), and the transverse coordinates are conveniently chosen as $\rho=\sqrt{x^{2}+y^{2}}$ and the azimuthal angle $\phi$. Ultrarelativistic kinematics in the beam direction suggests the longitudinal proper time $\tau=\sqrt{t^{2}-z^{2}}$ and the longitudinal space-time-rapidity $\eta=\operatorname{arth}(z / t)$ as appropriate longitudinal and temporal coordinates:

$$
R^{\mu}=(\tau \operatorname{ch} \eta, \rho \cos \phi, \rho \sin \phi, \tau \operatorname{sh} \eta) .
$$

The particle momenta are parametrized by their rapidity $Y=\operatorname{arth}\left(P_{z} / E\right)$ along the beam direction and their transverse mass $M_{t}=\sqrt{M^{2}+P_{t}^{2}}$ :

$$
P^{\mu}=\left(M_{t} \operatorname{ch} Y, P_{t} \cos \Phi, P_{t} \sin \Phi, M_{t} \operatorname{sh} Y\right) .
$$

The flow 4-velocity is conveniently parametrized in terms of longitudinal and transverse flow rapidities $\eta_{l}$ and $\eta_{t}$, respectively:

$$
u^{\mu}(R)=\left(\operatorname{ch} \eta_{l} \operatorname{ch} \eta_{t}, \operatorname{sh} \eta_{t} \cos \phi, \operatorname{sh} \eta_{t} \sin \phi, \operatorname{sh} \eta_{l} \operatorname{ch} \eta_{t}\right),
$$

where th $\eta_{i}=v_{i}, i=l$, $t$, defines the corresponding flow velocities. In the spirit of Bjorken [43] we assume a scaling velocity profile $v_{l}=z / t$ in the beam direction while taking a power-law rapidity profile in the transverse direction which is independent of $z$ and $t$ :

$$
\eta_{l}(\tau, \eta, \rho)=\eta, \quad \eta_{t}(\tau, \eta, \rho)=\eta_{f}\left(\frac{\rho}{\Delta \rho}\right)^{\alpha} .
$$

Here $\Delta \rho$ characterizes the transverse size of the fireball (see below), while $\eta_{f}$ represents the strength of the transverse flow; the power $\alpha$ of the transverse flow profile is generally chosen as $\alpha=1$, except for some tests with $\alpha=0.5$ and $\alpha=2$ as noted in the text.

As the fireball expands the scattering rate of the particles decreases until finally the thermalization of the system breaks down and the particles freeze out. Consistently with the above Ansatz for the expansion flow profile we assume that this happens at a fixed longitudinal proper time $\tau_{0}$ and set $\bar{H}(R)=H(\eta, \rho) \delta\left(\tau-\tau_{0}\right)$. For the longitudinal and transverse shape of the density profile $H(R)$ we take Gaussians with widths $\Delta \eta$ and $\Delta \rho$, respectively.

With these ingredients the distribution functions in (2.3) take the form

$$
\begin{aligned}
& f_{i}(R, P)=e^{\mu_{i} / T} e^{-P \cdot u(R) / T} H(R), \quad i=\mathrm{p}, \mathrm{n} \\
& H(R)=H(\eta, \rho)=\exp \left(-\frac{\rho^{2}}{2(\Delta \rho)^{2}}-\frac{\eta^{2}}{2(\Delta \eta)^{2}}\right) .
\end{aligned}
$$

The density profile is normalized to a total covariant freeze-out volume $V_{\text {cov }}$ :

$$
V_{\mathrm{cov}}=\int d^{4} R \bar{H}(R)=(2 \pi)^{\frac{3}{2}}(\Delta \rho)^{2}(\Delta \eta) \tau_{0},
$$

where $d^{4} R=\tau d \tau \rho d \rho d \eta d \phi$. For freeze-out at constant longitudinal proper time, the integration measure in $(2.3)$ over the freeze-out hypersurface is given by $P \cdot d^{3} \sigma(R)=$ $\tau_{0} M_{t} \rho d \rho \operatorname{ch}(\eta-Y) d \eta d \phi$.

\section{B. Non-zero emission duration}

In [9], instead of a Cooper-Frye integral over a 3-dim freeze-out hypersurface, invariant spectra are calculated as a space-time integral $\int d^{4} R S_{i}(R, P)$ over an emission function

$$
\begin{gathered}
S_{i}(R, P)=\frac{2 J_{i}+1}{(2 \pi)^{3}} M_{t} \operatorname{ch}(\eta-Y) e^{\left(\mu_{i}-P \cdot u(R)\right) / T} \\
\times \tilde{H}(\eta, \rho) J(\tau) .
\end{gathered}
$$

$[\tilde{H}(\eta, \rho)=\sqrt{(2 / \pi)} H(\eta, \rho)$ differs from (2.8) only by the normalization. The present choice is more convenient for us because it absorbs some constant terms in the cluster spectra below which would otherwise scale with the nucleon number. However, it affects the interpretation of the total fireball volume $\left(\tilde{V}_{\text {cov }}=\sqrt{2 / \pi} V_{\text {cov }}\right)$, and of the fugacity factor $\exp (\mu / T)$. In the case of Ref. [9], $\mu / T$ is the fugacity averaged over the fireball; in the present case it is the fugacity at $R=\left(\tau_{0}, \boldsymbol{R}=\mathbf{0}\right)$.]

The function $J(\tau)$ implements a smearing of the freezeout hypersurface around $\tau_{0}$; the choice in [9] is

$$
J(\tau)=\frac{1}{\Delta \tau \sqrt{2 \pi}} \exp \left(\frac{\left(\tau-\tau_{0}\right)^{2}}{2(\Delta \tau)^{2}}\right) .
$$

General conditions for $J(\tau)$ are $\int d \tau J(\tau)=1, \int d \tau \tau J(\tau)$ $=\tau_{0}$, and $\Delta \tau \ll \tau_{0}$. The last one ensures that one can treat $f_{i}$ and $H$ as $\tau$-independent, and that freezeout times $\tau<0$ play no physical role.

For single-hadron spectra $J(\tau)$ can be immediately integrated over, reducing the space-time integral over the emission function to the Cooper-Frye form (2.1) with (2.8). A non-zero duration of particle emission $(\Delta \tau>0)$ has, however, an effect on cluster formation and on other two-particle correlations. In [9], two-pion correlation data from $\mathrm{Pb}+\mathrm{Pb}$ collisions at the SPS were fitted with $\Delta \tau=1.5 \mathrm{fm} / c$, although with considerable uncertainty [44. This value appears to be small enough to be able to neglect the $\tau$-dependence of the parameters in $f_{i}$ and $H$; since estimates in 45 have shown that the effect of $\Delta \tau>0$ on cluster formation should then be small, we will continue to use the simpler Cooper-Frye formalism (2.3) also for cluster spectra.

\section{Cluster spectra from the model source}

Inserting the expressions from Sec. II A into Eq. (2.3) one is led to the following integral: 


$$
\begin{aligned}
& E \frac{d N_{A}}{d^{3} P}=\frac{2 J_{A}+1}{(2 \pi)^{3}} e^{\frac{\mu_{A}}{T}} \tau_{0} M_{t} \int d \eta \rho d \rho d \tilde{\phi} \operatorname{ch}(\eta-Y) \\
& \quad \times e^{-\frac{M_{t} \operatorname{ch}(\eta-Y) \operatorname{ch} \eta_{t}(\rho)-P_{t} \operatorname{sh} \eta_{t}(\rho) \cos \tilde{\phi}}{T}-\frac{A \rho^{2}}{2(\Delta \rho)^{2}}-\frac{A \eta^{2}}{2(\Delta \eta)^{2}}} .
\end{aligned}
$$

The integration over $\tilde{\phi}=\phi-\Phi$ can be done analytically, yielding a modified Bessel function, but this is not very helpful for further analytical progress. More useful is the following observation [2, 46, 47]: for fireball temperatures below the deconfinement temperature of about $150 \mathrm{MeV}$, the ratio $M_{t} / T \gtrsim 6 A$ in the exponent is large, and the integral will receive contributions only from narrow intervals $\eta$ and $\rho$. We may thus expand the hyperbolic cosine and sine terms in the exponent, keeping only the leading terms:

$$
\begin{aligned}
\operatorname{ch}(\eta-Y) \operatorname{ch} \eta_{t} & \approx 1+\frac{1}{2}(\eta-Y)^{2}+\frac{1}{2} \eta_{t}^{2} ; \\
\cos \tilde{\phi} \operatorname{sh} \eta_{t} & \approx \eta_{f} \frac{x}{\Delta \rho} .
\end{aligned}
$$

This decouples the longitudinal and transverse flow, and the $\eta$-integration can now be done analytically. Note that the "saddle point approximation" (2.13) becomes unreliable for large $P_{t}$ and/or $\eta_{f}$ [48]. The remaining integrations are easy and give

$$
\begin{aligned}
E & \frac{d N_{A}}{d^{3} P} \approx \frac{2 J_{A}+1}{(2 \pi)^{3}} \frac{V_{\mathrm{cov}} M_{t} e^{\frac{\mu_{A}-M_{t}}{T}}}{\left(\frac{M_{t}}{T} \eta_{f}^{2}+A\right) \sqrt{\frac{M_{t}}{T}(\Delta \eta)^{2}+A}} \\
& \times \cosh \left(\frac{A Y}{\frac{M_{t}}{T}(\Delta \eta)^{2}+A}\right) \exp \left(\frac{(\Delta \eta)^{2} / 2}{\frac{M_{t}}{T}(\Delta \eta)^{2}+A}\right) \\
& \times \exp \left(\frac{P_{t}^{2} \eta_{f}^{2} /\left(2 T^{2}\right)}{\frac{M_{t}}{T} \eta_{f}^{2}+A}-\frac{A Y^{2} M_{t} /(2 T)}{\frac{M_{t}}{T}(\Delta \eta)^{2}+A}\right) \\
\approx & \frac{2 J_{A}+1}{(2 \pi)^{3}} e^{\frac{\mu_{A}-M}{T}} M_{t} V_{\mathrm{eff}}\left(A, M_{t}\right) \\
& \times \exp \left(-\frac{M_{t}-M}{T^{*}}-\frac{A Y^{2}}{2(\Delta \eta)^{2}}\right)
\end{aligned}
$$

with

$$
V_{\text {eff }}\left(A, M_{t}\right)=\frac{(2 \pi)^{\frac{3}{2}}(\Delta \rho)^{2}(\Delta \eta) \tau_{0}}{\left(\frac{M_{t}}{T} \eta_{f}^{2}+A\right) \sqrt{\frac{M_{t}}{T}(\Delta \eta)^{2}+A}}
$$

and

$$
T^{*}=T+\frac{M}{A} \eta_{f}^{2} .
$$

In going from (2.14a to (2.14b) we replaced the terms in the second line on (2.14a) by 1 and assumed nonrelativistic transverse cluster velocities, $v_{t}=P_{t} / M_{t} \ll 1$. (Eq. (2.16) should therefore not be applied to pions [49]!). $V_{\text {eff }}\left(A, M_{t}\right)$ is the effective volume contributing to the emission of clusters with mass number $A$ and transverse mass $M_{t}$ [47 (see Sect. IIE). $T^{*}$ is the inverse slope ("effective temperature") of the transverse momentum spectrum.

\section{Slope of the $M_{t}$-spectrum}

The relation (2.16) (with $M / A$ replaced by the hadron mass $m$ ) was suggested by $\mathrm{Nu} \mathrm{Xu}$ et al. [50,51] as a basis for a systematic separation of collective flow $\left(\eta_{f}\right)$ from thermal motion $(T)$ using single-particle spectra. It has recently become very popular, especially since in heavy ion collisions at the AGS and SPS the measured $m_{t^{-}}$ spectra of most hadronic species seem to follow it quite nicely by showing, at sufficiently low $p_{t}$, inverse slope parameters which rise linearly with the hadron rest mass [5]. This has been interpreted as strong evidence for the existence of transverse collective flow [39,50,51].

When applied to nuclear clusters, however, Eq. (2.16) predicts exactly the same slope for all mass numbers $A$ since $M \sim A$. This is in contradiction with experiments both at the AGS and the SPS which show considerably flatter $m_{t}$-slopes for deuterons than for protons [52 54]. In order to understand the possible origins of this discrepancy one must remember that Eq. (2.16) rests not only on non-relativistic kinematics, but also on the Gaussian transverse density and linear transverse flow rapidity profiles. These were selected in [2, 3, 9, 44, 47] for technical convenience, and we have kept them here for ease of comparability. It was pointed out, however, by Polleri et al. [55] that the Gaussian density profile gives too much weight to the center of the fireball where the transverse flow is weak. Since according to Eqs. (2.8a) and (2.3) the profile function $H(\rho)$ enters the expression for nuclear cluster spectra with the $A^{\text {th }}$ power, the region of weak transverse flow receives the more weight the heavier the cluster; for Gaussian profiles this divides the effective strength of the transverse flow exactly by a factor $A$ [5.5] and thus causes the $A$-independence of the cluster slopes.

As we will show in Sect. VIIB this cannot be compensated by decreasing the power $\alpha$ in the transverse flow profile (2.7) even though this does lead to a more rapid increase of the transverse flow rapidity at small values of $\rho$. Phenomenological consistency with the observed $A$-dependence of the cluster $M_{t}$-slopes can only be achieved by selecting density profiles $H(\rho)$ which, when taken to the $A^{\text {th }}$ power, don't lose weight in the relevant region of large transverse flow. A transverse box profile (or a smooth version of it) satisfies this requirement and is shown to work well in Sect. VIIB.

The Gaussian transverse density profile is problematic also for a different reason: in combination with the transverse flow profile (2.7) it leads to acausal behaviour for heavy particles with large transverse velocities $v_{t}$. Due to the Boltzmann factor $\exp (-P \cdot u(R) / T)$ in the integrand of (2.1) or (2.3), which gives strong weight to space-time points $R$ with $u(R)=P / M$ (the more so the larger $M / T)$, such particles are emitted mostly from fluid cells at large transverse radii $\rho$, i.e. in the tail of the Gaussian density distribution. Such matter should not exist, however: a cold $\mathrm{Pb}$ nucleus with an rms radius $\rho_{\text {rms }}=5.5 \mathrm{fm}$ [56], corresponding to a hard sphere 
radius $\rho_{0}=\sqrt{5 / 3} \rho_{\mathrm{rms}}$, can expand in time $\tau_{0}$ at most to a maximum transverse radius of $\rho_{\max }=\rho_{0}+\tau_{0}$ (even less, if the transverse expansion velocity is $<c$ ). Thus, no matter should exist at radii $\rho>\rho_{\max }$. In our case nucleons with transverse velocities $v_{t}>0.6 c$ tend to be emitted from causally forbidden regions. (For the lighter pions the problem is much less severe due to the larger thermal smearing.)

For the Gaussian density profile $(2.8 \mathrm{~b})$ we will therefore restrict our attention to nucleons and clusters with $v_{t} \leq 0.6 c$, i.e. $1.0 \leq M_{t} / M \leq 1.25$. For a proper description of clusters with larger velocities the Gaussian transverse density profile must be modified, either by cutting it off by hand or by replacing it with a causally consistent box profile. Unfortunately, this forfeits the simple analytical expressions (2.14 2.16) and the direct comparability with the published results from HBT analyses of two-pion correlations [3,9,44].

As for the longitudinal rapidity spectrum of clusters, we expect from Eq. (2.14) for its width a decrease with $1 / \sqrt{A}$ compared to nucleons.

\section{E. Effective source volume $V_{\text {eff }}$ and relation to HBT}

The Boltzmann factor in (2.8a couples the particle momentum to the flow vector $u(R)$. This causes a correlation between the velocity and the spatial coordinates of the particle, with a "coupling constant" $M / T$ which increases with the particle mass. Particles inside the fireball are thus sorted with respect to their velocities, and particles of given momentum are localized in regions of the fireball where the flow velocity is close to the particle velocity.

Thus only a fraction of the total fireball volume $V_{\text {cov }}$ is able to emit particles with given momentum. It is this "homogeneity volume" $V_{\text {hom }}\left(m_{t}\right)$ which is accessible through HBT measurements [3]. The HBT radii $\mathcal{R}_{\|}\left(m_{t}\right)$ and $\mathcal{R}_{\perp}\left(m_{t}\right)$ which can be extracted from the 2 particle correlation function in the YKP parametrization [57 describe the corresponding longitudinal and transverse lengths of homogeneity in the source. They can be evaluated for the model (2.8) as space-time variances of the source using the general expressions given in [57]. If these variances are evaluated in the saddle-point approximation (2.13) one finds 46,57

$$
\begin{aligned}
& \mathcal{R}_{\perp}\left(m_{t}\right)=\frac{\Delta \rho}{\sqrt{1+\frac{m_{t}}{T} \eta_{f}^{2}}}, \\
& \mathcal{R}_{\|}\left(m_{t}\right)=\frac{\tau_{0} \Delta \eta}{\sqrt{1+\frac{m_{t}}{T}(\Delta \eta)^{2}}} .
\end{aligned}
$$

These are just the factors occurring in the effective volume 2.15) for $A=1$ :

$$
\begin{aligned}
V_{\mathrm{eff}}\left(1, m_{t}\right) & =(2 \pi)^{3 / 2} \mathcal{R}_{\|}\left(m_{t}\right) \mathcal{R}_{\perp}^{2}\left(m_{t}\right) \\
& \equiv(2 \pi)^{3 / 2} V_{\mathrm{hom}}\left(m_{t}\right)
\end{aligned}
$$

Thus the effective volume $V_{\text {eff }}$ in the cluster spectrum (2.14) is very closely related to the homogeneity volume extracted from HBT measurements with pairs of identical hadrons:

$$
V_{\mathrm{eff}}\left(A, M_{t}\right)=\frac{V_{\mathrm{eff}}\left(1, m_{t}\right)}{A^{3 / 2}}=\left(\frac{2 \pi}{A}\right)^{3 / 2} V_{\mathrm{hom}}\left(m_{t}\right) .
$$

For deuterons this implies an effective volume which is about $1 / 3$ that of the nucleons.

Since at AGS and SPS energies $\Delta \eta \gtrsim 1$ (see Sect. IIF), the longitudinal flow term $\sim\left(m_{t} / T\right)(\Delta \eta)^{2}$ in $\mathcal{R}_{\|}$dominates over the geometric term $\sim 1$. In the transverse direction the flow term $\sim\left(m_{t} / T\right) \eta_{f}^{2}$ is much smaller, and the two terms compete with each other, depending on the value of $\eta_{f}$. Higher temperature increases, larger transverse flow decreases the lengths of homogeneity. For fixed $T, \eta_{f}$ the homogeneity lengths decrease with increasing transverse mass $m_{t}$. According to Eqs. 2.18,2.19) this implies for fixed particle momenta a decrease of $V_{\text {eff }}\left(m_{t}\right)$ with the particle rest mass roughly like $M^{-3 / 2}$. Due to the non-negligible geometric contribution in $\mathcal{R}_{\perp}$ the decrease is actually somewhat weaker; for realistic parameters (see below) the combination $M_{t} V_{\text {eff }}$ in $(2.14 \mathrm{~b})$ turns out to be practically independent of $M_{t}: M_{t} V_{\text {eff }}\left(A, M_{t}\right) \approx M V_{\text {eff }}(A, M)$.

Eqs. (2.17 2.19) indicate perfect $m_{t}$-scaling of the HBT radii and the effective volume. If true, the values of $V_{\text {eff }}$ to be used in $2.14 \mathrm{~b}$ for nuclear clusters could be directly extracted from two-pion HBT measurements at very high $p_{t}$ such that the transverse mass $M_{t}$ would be the same. Unfortunately, this is an artifact of the saddle-point approximation (2.13) 48]; a numerical evaluation shows that $m_{t}$-scaling of the HBT radii is broken by transverse flow, albeit weakly [57,3]. Appropriate care must thus be taken before using (2.18) to compare the effective volume $V_{\text {eff }}$ in cluster formation with the homogeneity volume $V_{\text {hom }}$ extracted from HBT measurements with pions or kaons.

\section{F. Parameters for central $\mathrm{Pb}+\mathrm{Pb}$ collisions}

We close this section by specifying the model parameters to be used later in the calculation of cluster yields and spectra. In $\mathrm{Pb}+\mathrm{Pb}$ collisions at $158 \mathrm{GeV}$ per nucleon, mid-rapidity is at $y_{\mathrm{cm}}=2.91$. For very central collisions (4-5\% of the total inelastic cross section $\sigma_{\text {tot }}^{\text {inel }}$, corresponding to impact parameters $b \leq 3.5 \mathrm{fm}$ ), the analysis of pion spectra and two-pion correlations from NA49 10,58,59 led to the following estimates for the model parameters [9,44]: $\Delta \eta \approx 1.3, \Delta \rho \approx 7 \mathrm{fm}, \tau_{0} \approx 9 \mathrm{fm} / c$, $\Delta \tau \approx 1.5 \mathrm{fm} / c, \eta_{f} \approx 0.35$ and $T \approx 130 \mathrm{MeV}$. To estimate the effects of possible errors in $T$ and $\eta_{f}$, we will additionally test the $\left(T, \eta_{f}\right)$ combinations of $(100 \mathrm{MeV}$, $0.43)$ and $(168 \mathrm{MeV}, 0.28)$, which also describe the slope of the single particle $m_{t}$-spectrum of negatively charged hadrons (mainly pions), but not the behaviour of the 
transverse HBT radius $\mathcal{R}_{\perp}^{\pi}\left(m_{t}\right)$. The effects of uncertainties in the determination of $\tau_{0}$ and $\Delta \rho$, which may also be important, have not been studied. From the above parameter values we calculate a total covariant fireball volume (2.9) of $V_{\text {cov }} \approx 9000 \mathrm{fm}^{3}$, homogeneity lengths of $\mathcal{R}_{\|}(1 \mathrm{GeV}) \approx(3.2 \pm 0.4)$ fm and $\mathcal{R}_{\perp}(1 \mathrm{GeV}) \approx$ $(5.1 \pm 0.8) \mathrm{fm}$, and an effective volume for nucleons of $V_{\mathrm{eff}}(1, m) \approx(0.15 \pm 0.06) \times V_{\mathrm{cov}}$.

\section{THE QUANTUM MECHANICS OF COALESCENCE}

\section{A. Reference frames}

So far we have treated nuclear clusters as point-like particles without internal structure. This is not realistic: the deuteron, for example, has already an rms radius of almost $2 \mathrm{fm}[60]$ which is not much smaller than the homogeneity radii given above. A proper inclusion of finite size effects and the internal cluster wave function in the description of the coalescence process is therefore mandatory. This requires a quantum mechanical treatment. In this section we will concentrate on the two-body problem, i.e. the coalescence of (anti)deuterons.

Due to its small binding energy, only nucleons with small relative momentum can form a deuteron. In the deuteron rest frame (d-frame) the relative motion of the two nucleons and their coalescence into a bound state are thus described by non-relativistic quantum mechanics. A relativistic formulation is needed, on the other hand, to describe the motion of the deuterons (i.e. of the center of mass of the nucleon pair) in the fireball rest frame (f-frame).

In the f-frame, we denote the deuteron's momentum and c.m. space-time coordinates by $P_{\mathrm{d}}=\left(E_{\mathrm{d}}, \boldsymbol{P}_{\mathrm{d}}\right)$ and $R_{\mathrm{d}}=\left(R_{\mathrm{d}}^{0}, \boldsymbol{R}_{\mathrm{d}}\right)$, and the momenta and space-time coordinates of its two nucleons by $P_{ \pm}$and $R_{ \pm}=\left(R_{ \pm}^{0}, \boldsymbol{R}_{ \pm}\right)$. In the d-frame, the four-momenta $q_{ \pm}$of the two nucleons are given in terms of their relative momentum $\boldsymbol{q}$ by

$$
q_{ \pm}^{\mu}=\left(\sqrt{m^{2}+\boldsymbol{q}^{2}}, \pm \boldsymbol{q}\right) .
$$

Their space-time coordinates $r_{ \pm}=\left(t_{\mathrm{d}}, \boldsymbol{r}_{ \pm}\right)$in the dframe can be expressed in terms of the d-frame c.m. coordinates $r_{\mathrm{d}}=\left(t_{\mathrm{d}}, \boldsymbol{r}_{\mathrm{d}}\right)$ and their relative distance $r^{\nu}=(0, \boldsymbol{r})$ as $r_{ \pm}=r_{\mathrm{d}} \pm \frac{1}{2} r$. Note that in the d-frame $r$ has a vanishing zero component, consistent with the equal-time nature of non-relativistic quantum mechanics. The deuteron's four-velocity in the f-frame we denote by $b^{\lambda}=\left(b^{0}, \boldsymbol{b}\right)=P_{\mathrm{d}}^{\lambda} / m_{\mathrm{d}}$. The matrix $L$ for a boost from the $\mathrm{f}$-frame into the $\mathrm{d}$-frame is then given by

$$
L^{\mu \nu}=\left(\begin{array}{cccc}
b_{0} & b_{x} & b_{y} & b_{z} \\
-b_{x} & -1-\frac{b_{x}^{2}}{1+b_{0}} & -\frac{b_{x} b_{y}}{1+b_{0}} & -\frac{b_{x} b_{z}}{1+b_{0}} \\
-b_{y} & -\frac{b_{x} b_{y}}{1+b_{0}} & -1-\frac{b_{y}}{1+b_{0}} & -\frac{b_{y} b_{z}}{1+b_{0}} \\
-b_{z} & -\frac{b_{x} b_{z}}{1+b_{0}} & -\frac{b_{y} b_{z}}{1+b_{0}} & -1-\frac{b_{z}^{2}}{1+b_{0}}
\end{array}\right)
$$

The matrix $L^{-1}$ for the inverse boost follows by substituting $b_{i} \leftrightarrow-b_{i}$ for $i=x, y, z$.

We consider the f-frame coordinates $P_{ \pm}$and $R_{ \pm}$as functions of the f-frame c.m. coordinates $P_{\mathrm{d}}$ and $R_{\mathrm{d}}$ and the d-frame relative coordinates $q$ and $r$ :

$$
\begin{aligned}
R_{ \pm}^{\mu} & =\left(L^{-1}\right)^{\mu \nu} r_{ \pm, \nu}=R_{\mathrm{d}}^{\mu} \pm \frac{1}{2}\left(L^{-1}\right)^{\mu \nu} r_{\nu}, \\
P_{ \pm}^{\mu} & =\left(L^{-1}\right)^{\mu \nu} q_{ \pm, \nu} .
\end{aligned}
$$

For the internal (relative) wave function $\varphi_{\mathrm{d}}$ of the deuteron we will consider both the Hulthen form with the parameters $\alpha=0.23 \mathrm{fm}^{-1}, \beta=1.61 \mathrm{fm}^{-1}$, and the wave function of a spherical harmonic oscillator with the size parameter $d=3.2 \mathrm{fm}(r \equiv|\boldsymbol{r}|)$ :

$$
\begin{aligned}
& \varphi_{\mathrm{d}}(\boldsymbol{r})=\sqrt{\frac{\alpha \beta(\alpha+\beta)}{2 \pi(\alpha-\beta)^{2}}} \frac{e^{-\alpha r}-e^{-\beta r}}{r}, \\
& \varphi_{\mathrm{d}}(\boldsymbol{r})=\left(\pi d^{2}\right)^{-3 / 4} \exp \left(-\frac{r^{2}}{2 d^{2}}\right) .
\end{aligned}
$$

While the first choice gives a better description of the deuteron ground state, the second one is technically advantageous since it allows for a largely analytic evaluation of the coalescence factor below. Both wave functions reproduce the measured rms radius of $1.96 \mathrm{fm}$ 60. Please note that the variable $\boldsymbol{r}$ describes the diameter and not the radius of the relative wave functions such that the $\mathrm{rms}$ radius is given by $r_{\mathrm{rms}}^{2}=\int d^{3} r\left(\frac{r}{2}\right)^{2}\left|\varphi_{\mathrm{d}}(\boldsymbol{r})\right|^{2}$.

\section{B. The density matrix formalism}

According to the rules of statistical quantum mechanics [61] the number of created deuterons with momentum $P_{\mathrm{d}}$ is given by projecting the deuteron density matrix onto the two-nucleon density matrix in the fireball at the freeze-out time $t_{\mathrm{f}}$ :

$$
\begin{aligned}
\frac{d N_{\mathrm{d}}}{d^{3} P_{\mathrm{d}}} & \sim \frac{1}{2 !} \int d^{3} x_{1} d^{3} x_{2} d^{3} x_{1}^{\prime} d^{3} x_{2}^{\prime} \phi_{\mathrm{d}}^{*}\left(\boldsymbol{x}_{1}, \boldsymbol{x}_{2}\right) \phi_{\mathrm{d}}\left(\boldsymbol{x}_{1}^{\prime}, \boldsymbol{x}_{2}^{\prime}\right) \\
& \times\left\langle\psi^{\dagger}\left(\boldsymbol{x}_{2}^{\prime}, t_{\mathrm{f}}\right) \psi^{\dagger}\left(\boldsymbol{x}_{1}^{\prime}, t_{\mathrm{f}}\right) \psi\left(\boldsymbol{x}_{1}, t_{\mathrm{f}}\right) \psi\left(\boldsymbol{x}_{2}, t_{\mathrm{f}}\right)\right\rangle .
\end{aligned}
$$

The correct spin and isospin factors will be added later after introducing Wigner functions. A rigorous ansatz with projection operators that select the correct spin and isospin state for the deuteron from the two-particle density matrix can be found in 29 .

In the detector the deuteron is observed as a free momentum eigenstate. Its wave function is therefore given 
by a plane wave for the c.m. motion multiplied by the internal wave function $\varphi_{\mathrm{d}}: \phi_{\mathrm{d}}\left(\boldsymbol{x}_{1}, \boldsymbol{x}_{2}\right)=(2 \pi)^{-3 / 2} \exp \left(i \boldsymbol{P}_{\mathrm{d}}\right.$. $\left.\left(\boldsymbol{x}_{1}+\boldsymbol{x}_{2}\right) / 2\right) \varphi_{\mathrm{d}}\left(\boldsymbol{x}_{1}-\boldsymbol{x}_{2}\right)$. The two-nucleon density matrix in the fireball is not known and has to be approximated. We assume that at freeze-out the nucleons are uncorrelated:

$$
\begin{aligned}
& \left\langle\psi^{\dagger}\left(\boldsymbol{x}_{2}^{\prime}, t\right) \psi^{\dagger}\left(\boldsymbol{x}_{1}^{\prime}, t\right) \psi\left(\boldsymbol{x}_{1}, t\right) \psi\left(\boldsymbol{x}_{2}, t\right)\right\rangle \\
& =\left\langle\psi^{\dagger}\left(\boldsymbol{x}_{2}^{\prime}, t\right) \psi\left(\boldsymbol{x}_{2}, t\right)\right\rangle\left\langle\psi^{\dagger}\left(\boldsymbol{x}_{1}^{\prime}, t\right) \psi\left(\boldsymbol{x}_{1}, t\right)\right\rangle .
\end{aligned}
$$

The one-particle density matrix can be expressed through the 1-body Wigner function as 661,62

$$
\begin{aligned}
& \left\langle\psi^{\dagger}\left(\boldsymbol{x}^{\prime}, t\right) \psi(\boldsymbol{x}, t)\right\rangle \\
& \quad=\int \frac{d^{3} p}{(2 \pi)^{3}} f^{\mathrm{W}}\left(\boldsymbol{p} ; t, \frac{\boldsymbol{x}+\boldsymbol{x}^{\prime}}{2}\right) \exp \left(i \boldsymbol{p} \cdot\left(\boldsymbol{x}-\boldsymbol{x}^{\prime}\right)\right) .
\end{aligned}
$$

To evalute the integral (3.5), we introduce new coordinates $\boldsymbol{r}_{+}=\frac{1}{2}\left(\boldsymbol{x}_{1}+\boldsymbol{x}_{1}^{\prime}\right), \boldsymbol{r}_{-}=\frac{1}{2}\left(\boldsymbol{x}_{2}+\boldsymbol{x}_{2}^{\prime}\right), \boldsymbol{\xi}=\boldsymbol{x}_{1}-\boldsymbol{x}_{1}^{\prime}$ $-\left(\boldsymbol{x}_{2}-\boldsymbol{x}_{2}^{\prime}\right)$ and $\boldsymbol{\rho}=\frac{1}{2}\left(\boldsymbol{x}_{1}-\boldsymbol{x}_{1}^{\prime}+\boldsymbol{x}_{2}-\boldsymbol{x}_{2}^{\prime}\right)$. The first two are the classical coordinates of the coalescing nucleons and will be further reexpressed by $\boldsymbol{r}_{\mathrm{d}}$ and $\boldsymbol{r}$. For the momentum vectors $\boldsymbol{p}_{1}$ and $\boldsymbol{p}_{2}$ introduced via (3.7) we define the relative momentum $\boldsymbol{q}=\frac{1}{2}\left(\boldsymbol{p}_{1}-\boldsymbol{p}_{2}\right)$ and the total momentum $\boldsymbol{P}_{\mathrm{d}}=\boldsymbol{p}_{1}+\boldsymbol{p}_{2}$; the identification with the deuteron's momentum is due a corresponding $\delta$ function for 3-momentum conservation which arises from the $d^{3} \rho$ integration. The $d^{3} \xi$-integration leads to a term which we recognize as the Wigner transform of the internal deuteron wave function,

$$
\mathcal{D}(\boldsymbol{r}, \boldsymbol{q})=\int d^{3} \xi e^{-i \boldsymbol{q} \cdot \boldsymbol{\xi}} \varphi_{\mathrm{d}}\left(\boldsymbol{r}+\frac{\boldsymbol{\xi}}{2}\right) \varphi_{\mathrm{d}}^{*}\left(\boldsymbol{r}-\frac{\boldsymbol{\xi}}{2}\right),
$$

which has the following normalization property:

$$
\int d^{3} r \int \frac{d^{3} q}{(2 \pi)^{3}} \mathcal{D}(\boldsymbol{r}, \boldsymbol{q})=\int d^{3} r \varphi_{\mathrm{d}}^{*}(\boldsymbol{r}) \varphi_{\mathrm{d}}(\boldsymbol{r})=1 .
$$

For the deuteron spectrum we thus obtain

$$
\begin{gathered}
\frac{d N_{\mathrm{d}}}{d^{3} P_{\mathrm{d}}}=\frac{3}{(2 \pi)^{3}} \int d^{3} r_{\mathrm{d}} \int \frac{d^{3} q d^{3} r}{(2 \pi)^{3}} \mathcal{D}(\boldsymbol{r}, \boldsymbol{q}) \\
\times f_{\mathrm{p}}^{\mathrm{W}}\left(q_{+}, r_{+}\right) f_{\mathrm{n}}^{\mathrm{W}}\left(q_{-}, r_{-}\right),
\end{gathered}
$$

where $q_{ \pm}, r_{ \pm}$are functions of the integration variables according to Eqs. (3.3).

\section{The problem of energy conservation}

Since in Eq. (3.5) the two-nucleon density matrix is projected on the density matrix of an energy eigenstate (the free deuteron), energy is clearly conserved. On the other hand, if we were to replace the Wigner functions in (3.10) by classical distribution functions of on-shell nucleons (as done e.g. in 21]) we would violate energy conservation: due to the deuteron binding energy two free nucleons cannot coalesce into a deuteron without help of a third body. In other words, one or both of the Wigner functions in (3.10) are probed off-shell, and the nucleons in this expression cannot be considered as free particles. (For free distinguishable particles in a thermalized system the Wigner function is identical to the Boltzmann distribution [61.)

A formalism which makes the conservation of energy during coalescence more explicit by including the scattering with a third body and which thereby gives more control over the replacement of the Wigner functions by classical distributions was developed by Danielewicz and Schuck 23]. Instead of (3.5) they start from 63.

$$
\begin{aligned}
& \frac{d N_{\mathrm{d}}}{d^{3} P_{\mathrm{d}}}=\lim _{T, T^{\prime} \rightarrow \infty} \frac{1}{T T^{\prime}} \frac{1}{2 !} \int_{T}^{2 T} d t \int_{T^{\prime}}^{2 T^{\prime}} d t^{\prime} \int d^{3} x_{1} d^{3} x_{2} \\
& \quad \times d^{3} x_{1}^{\prime} d^{3} x_{2}^{\prime} e^{i E_{\mathrm{d}}\left(t-t^{\prime}\right)} \phi_{\mathrm{d}}^{*}\left(\boldsymbol{x}_{1}, \boldsymbol{x}_{2}\right) \phi_{\mathrm{d}}\left(\boldsymbol{x}_{1}^{\prime}, \boldsymbol{x}_{2}^{\prime}\right) \\
& \quad \times\left\langle\psi^{\dagger}\left(\boldsymbol{x}_{2}^{\prime}, t^{\prime}\right) \psi^{\dagger}\left(\boldsymbol{x}_{1}^{\prime}, t^{\prime}\right) \psi\left(\boldsymbol{x}_{1}, t\right) \psi\left(\boldsymbol{x}_{2}, t\right)\right\rangle .
\end{aligned}
$$

They then consider the equations of motion for the correlator $\left\langle\psi^{\dagger}\left(\boldsymbol{x}_{2}^{\prime}, t^{\prime}\right) \psi^{\dagger}\left(\boldsymbol{x}_{1}^{\prime}, t^{\prime}\right) \psi\left(\boldsymbol{x}_{1}, t\right) \psi\left(\boldsymbol{x}_{2}, t\right)\right\rangle$. These include interactions with third particles which can put one of the two nucleons slightly off-shell. This nucleon can then form a deuteron with an on-shell nucleon without violating energy conservation. It is convenient at this point to introduce a four-vector notation, with momentum four-vectors which can be either off-shell (indicated by an asterisk) or on-shell (no asterisk): $p_{i}=\left(E_{i}, \boldsymbol{p}_{i}\right)$, $p_{i}^{*}=\left(\omega, \boldsymbol{p}_{i}\right)$, and $r_{i}=\left(t_{i}, \boldsymbol{r}_{i}\right)$. For the evaluation of (3.11) we refer to Ref. [23]; the result is (see Eqs. $(16,19)$ in $223 \mid$ )

$$
\begin{aligned}
& \frac{d N_{\mathrm{d}}}{d^{3} P_{\mathrm{d}}}=\frac{-3 i}{(2 \pi)^{3}} \int d^{4} r_{\mathrm{d}} d^{3} r \int \frac{d^{4} p_{1}}{(2 \pi)^{4}} \frac{d^{3} p_{2}}{(2 \pi)^{3}} \\
& \quad \times(2 \pi)^{4} \delta^{4}\left(P_{\mathrm{d}}-p_{1}^{*}-p_{2}\right) \mathcal{D}\left(\boldsymbol{r}, \frac{\boldsymbol{p}_{1}-\boldsymbol{p}_{2}}{2}\right) \\
& \quad \times\left(\Sigma_{\mathrm{p}}^{<}\left(p_{1}^{*}, r_{+}\right) f_{\mathrm{n}}^{\mathrm{W}}\left(p_{2}, r_{-}\right)+\Sigma_{\mathrm{n}}^{<}\left(p_{1}^{*}, r_{+}\right) f_{\mathrm{p}}^{\mathrm{W}}\left(p_{2}, r_{-}\right)\right),
\end{aligned}
$$

where the terms $\Sigma_{\mathrm{p}}^{<} f_{\mathrm{n}}^{\mathrm{W}}$ and $\Sigma_{\mathrm{n}}^{<} f_{\mathrm{p}}^{\mathrm{W}}$ give equal contributions. The rate $\Sigma_{\mathrm{N}}^{<}\left(p^{*}, x\right)$ for producing a nucleon with off-shell four-momentum $p^{*}$ at point $x$ is given by the scattering of the nucleon with a third particle:

$$
\begin{gathered}
-i \Sigma_{\mathrm{N}}^{<}\left(p^{*}, x\right)=\sum_{j} \int \frac{d^{3} q}{(2 \pi)^{3}} \frac{d^{3} p^{\prime}}{(2 \pi)^{3}} \frac{d^{3} q^{\prime}}{(2 \pi)^{3}} \\
\quad \times(2 \pi)^{4} \delta^{4}\left(p^{*}+q-p^{\prime}-q^{\prime}\right)\left|M_{\mathrm{N} j \rightarrow \mathrm{N} j}\right|^{2} \\
\quad \times f_{\mathrm{N}}^{\mathrm{W}}\left(p^{\prime}, x\right) f_{j}^{\mathrm{W}}\left(q^{\prime}, x\right)\left(1 \pm f_{j}^{\mathrm{W}}(q, x)\right) .
\end{gathered}
$$

Ref. 23 considers a gas of nucleons only, and therefore there is no summation over particle species $j$ as in (3.13). Our fireball, however, mainly consists of pions, and all particle species with large cross sections with nucleons must be accounted for in the production rate.

With the 4-dimensional $\delta$-functions in $3.12,3.13$ now explicitly accounting also for energy conservation, we proceed to approximate the Wigner distributions $f_{i}^{\mathrm{W}}$ in 
these equations by the classical thermal distribution functions (2.8). Using the momentum-conserving $\delta$-function $\delta^{4}\left(p^{*}+q-p^{\prime}-q^{\prime}\right)$ we then have the identity

$$
\begin{aligned}
& \frac{e^{-u \cdot p^{\prime} / T}}{e^{u \cdot q^{\prime} / T} \mp 1}\left(1 \pm \frac{1}{e^{u \cdot q / T} \mp 1}\right) \\
& =\frac{e^{-u \cdot p^{*} / T}}{e^{u \cdot q / T} \mp 1}\left(1 \pm \frac{1}{e^{u \cdot q^{\prime} / T} \mp 1}\right)
\end{aligned}
$$

and hence

$$
\begin{gathered}
-i \Sigma_{\mathrm{N}}^{<}\left(p^{*}, x\right) \approx f_{\mathrm{N}}\left(p^{*}, x\right) \sum_{j} \int \frac{d^{3} q}{(2 \pi)^{3}} f_{j}(q, x) \\
\times\left[\int \frac{d^{3} p^{\prime}}{(2 \pi)^{3}} \frac{d^{3} q^{\prime}}{(2 \pi)^{3}}(2 \pi)^{4} \delta^{4}\left(p^{*}+q-p^{\prime}-q^{\prime}\right)\right. \\
\left.\times\left|M_{\mathrm{N} j \rightarrow \mathrm{N} j}\right|^{2}\left(1 \pm f_{j}\left(q^{\prime}, x\right)\right)\right]
\end{gathered}
$$

The term in square brackets is the scattering cross section times the relative velocity between (off-shell) nucleons of momentum $\boldsymbol{p}$ and particles $j$ with momentum $\boldsymbol{q}$, averaged over the thermal distributions: $[\ldots]=\left\langle\sigma_{\mathrm{N} j} v_{\mathrm{N} j}(\boldsymbol{p}, \boldsymbol{q})\right\rangle$. The remaining integral over $d^{3} q$ and summation over $j$ then gives the total scattering rate or inverse scattering time of a nucleon with momentum $\boldsymbol{p}$ at point $x$ :

$$
-i \Sigma_{\mathrm{N}}^{<}\left(p^{*}, x\right)=\frac{f_{\mathrm{N}}\left(p^{*}, x\right)}{\tau_{\mathrm{sca}}^{\mathrm{N}}(\boldsymbol{p}, x)} .
$$

The production rate $\Sigma_{\mathrm{N}}^{<}\left(p^{*}, R\right)$ is therefore just given by the (off-shell!) nucleon distribution function $f_{\mathrm{N}}\left(p^{*}, R\right)$ divided by the scattering time of nucleons.

A deuteron has twice the scattering rate of its constituent nucleons. Since a scattering event is likely to break up the deuteron, and since we have by assumption free streaming particles after freeze-out at $t_{\mathrm{f}}$, the time integration $d t_{\mathrm{d}}$ in (3.12) should start at $t_{\mathrm{f}}-\tau_{\mathrm{scat}}^{\mathrm{N}} / 2$ and end at $t_{\mathrm{f}}$. Further assuming that we may treat the distribution functions as constant over this time interval, the inverse scattering time in (3.16) cancels against the time integration, and we are left with

$$
\begin{gathered}
\frac{d N_{\mathrm{d}}}{d^{3} P_{\mathrm{d}}}=\frac{3}{(2 \pi)^{3}} \int d^{3} r_{\mathrm{d}} \int \frac{d^{3} r d^{3} q}{(2 \pi)^{3}} \mathcal{D}(\boldsymbol{r}, \boldsymbol{q}) \\
\times f_{\mathrm{p}}\left(q_{+}, r_{+}\right) f_{\mathrm{n}}\left(q_{-}^{*} ; r_{-}\right) .
\end{gathered}
$$

The difference between this expression and $(3.10)$ is that the Wigner distributions have been replaced by classical distribution functions (2.8) one of which is off-shell $\left(q_{-}^{*}\right)$ so that a deuteron may be formed without violating energy conservation. The energy component of $q_{-}^{*}$ follows from the condition $q_{+}+q_{-}^{*}=\left(m_{\mathrm{d}}, \mathbf{0}\right)$ implied by the $\delta$-function in (3.12):

$$
\begin{aligned}
q_{+}^{\mu} & =\left(\sqrt{m^{2}+\boldsymbol{q}^{2}}, \boldsymbol{q}\right), \\
q_{-}^{* \mu} & =\left(m_{\mathrm{d}}-\sqrt{m^{2}+\boldsymbol{q}^{2}},-\boldsymbol{q}\right) .
\end{aligned}
$$

Eq. (3.17) is also a good basis for studying the quantitative effects of energy conservation on the coalescence process, for example by replacing the off-shell momentum $q_{-}^{*}$ by its on-shell limit.

\section{Coalescence in the highly relativistic fireball}

We now return to the relativistic motion of the particles in the fireball frame. In (3.10) or (3.17) we need the arguments of $f(p, x)$ in the f-frame, so we substitute $r_{ \pm}$and $q_{ \pm}$by $R_{ \pm}$and $P_{ \pm}$using (3.3). After performing the $d^{3} r$ and $d^{3} q$ integrations in the d-frame, we reexpress the $d^{3} r_{\mathrm{d}}$ integration over the deuteron's c.m. coordinate at time $t_{\mathrm{f}}$ in the d-frame in covariant form: $E_{\mathrm{d}} d^{3} r_{\mathrm{d}}=P_{\mathrm{d}} \cdot d^{3} \sigma\left(R_{\mathrm{d}}\right)$. The integral $d^{3} \sigma\left(R_{\mathrm{d}}\right)$ extends over the freeze-out surface $\Sigma_{\mathrm{f}}$ which is characterized by a relation $R_{\mathrm{d}, \mathrm{f}}^{0}\left(\boldsymbol{R}_{\mathrm{d}}\right)$ between the deuteron freeze-out time and point in the f-frame. In this way we obtain

$$
\begin{aligned}
E_{\mathrm{d}} \frac{d N_{\mathrm{d}}}{d^{3} P_{\mathrm{d}}} & =\frac{3}{(2 \pi)^{3}} \int_{\Sigma_{\mathrm{f}}} P_{\mathrm{d}} \cdot d^{3} \sigma\left(R_{\mathrm{d}}\right) \\
& \times f_{\mathrm{p}}\left(R_{\mathrm{d}}, \frac{P_{\mathrm{d}}}{2}\right) f_{\mathrm{n}}\left(R_{\mathrm{d}}, \frac{P_{\mathrm{d}}}{2}\right) \mathcal{C}_{\mathrm{d}}\left(R_{\mathrm{d}}, P_{\mathrm{d}}\right)
\end{aligned}
$$

with

$$
\mathcal{C}_{\mathrm{d}}\left(R_{\mathrm{d}}, P_{\mathrm{d}}\right)=\int \frac{d^{3} q d^{3} r}{(2 \pi)^{3}} \mathcal{D}(\boldsymbol{r}, \boldsymbol{q}) \frac{f\left(R_{+}, P_{+}\right) f\left(R_{-}, P_{-}\right)}{f^{2}\left(R_{\mathrm{d}}, \frac{P_{\mathrm{d}}}{2}\right)} .
$$

Up to the quantum mechanical correction factor $\mathcal{C}_{\mathrm{d}}$ this is identical with the classical formula (2.3). $\mathcal{C}_{\mathrm{d}}\left(R_{\mathrm{d}}, P_{\mathrm{d}}\right)$ is an integral over the internal phase-space coordinates and provides a measure for the homogeneity of the nucleon phase-space around the deuteron c.m. coordinates $\left(R_{\mathrm{d}}, P_{\mathrm{d}} / 2\right)$. For a homogeneous nucleon phase-space (static and very large systems) the second factor under the integral (3.20) (which we will call "homogeneity factor") approaches unity and (3.20) reduces to the normalization integral (3.9), yielding $\mathcal{C}_{\mathrm{d}}=1$.

As we will see, the $q$-dependence of the distribution functions $f\left(R_{+}, P_{+}\right)$and $f\left(R_{-}, P_{-}\right)$in (3.20) is much weaker than that of the deuteron Wigner density $\mathcal{D}(\boldsymbol{r}, \boldsymbol{q})$ which is peaked near $\boldsymbol{q}=0$. The distribution functions can thus be pulled outside the $q$-integral, yielding

$$
\mathcal{C}_{\mathrm{d}}\left(R_{\mathrm{d}}, P_{\mathrm{d}}\right) \approx \int d^{3} r \frac{f\left(R_{+}, \frac{P_{\mathrm{d}}}{2}\right) f\left(R_{-}, \frac{P_{\mathrm{d}}}{2}\right)}{f^{2}\left(R_{\mathrm{d}}, \frac{P_{\mathrm{d}}}{2}\right)}\left|\varphi_{\mathrm{d}}(\boldsymbol{r})\right|^{2} .
$$

This expression is now very similar to the Hagedorn model for cluster production in $p p$ collisions [37]. According to Hagedorn, the probability of finding a cluster with certain quantum numbers (mass $M$, spin, etc.) is equal to the probability to find a really elementary particle of the same quantum numbers, times the probability 
$\mathcal{C}$ that the cluster is contained inside the reaction volume $\Omega$ :

$$
\mathcal{C}=\int_{\Omega}|\varphi|^{2} d V
$$

For $p p$ collisions Hagedorn assumed [37] that the reaction volume was given by the pion Compton wavelength, $\Omega=4 \pi /\left(3 m_{\pi}^{3}\right)$, resulting in $\mathcal{C} \approx 0.17$ for the deuteron and $\mathcal{C} \approx 0.28$ for ${ }^{3} \mathrm{He}$ or ${ }^{3} \mathrm{H}$. This is consistent with the homogeneity radii extracted from two-pion HBT correlations in $p p$ collisions which are also of the order of the pion Compton wavelength 64.

\section{THE QUANTUM MECHANICAL CORRECTION FACTOR}

In this section we evaluate the coalescence factor $\mathcal{C}_{\mathrm{d}}$ for the source model discussed in Sec. II and relate it to the homogeneity radii $\mathcal{R}_{i}$ which can be extracted from HBT measurements.

\section{A. Integration over relative momenta}

The explicit dependence of the coordinates $R_{ \pm}$in (3.20) on $\boldsymbol{r}$ is given by (3.3):

$$
R_{ \pm}^{0}=R_{\mathrm{d}}^{0} \pm \frac{1}{2} \boldsymbol{b} \cdot \boldsymbol{r}, \boldsymbol{R}_{ \pm}=\boldsymbol{R}_{\mathrm{d}} \pm \frac{1}{2}\left(\boldsymbol{r}+\frac{\boldsymbol{b} \cdot \boldsymbol{r}}{1+b^{0}} \boldsymbol{b}\right) .
$$

From $R_{ \pm}$one must calculate

$$
\begin{aligned}
& \eta_{ \pm}=\frac{1}{2} \ln \frac{R_{ \pm}^{0}+R_{ \pm z}}{R_{ \pm}^{0}-R_{ \pm z}} \\
& \rho_{ \pm}=\sqrt{R_{ \pm x}^{2}+R_{ \pm y}^{2}} \\
& \tau_{ \pm}=\sqrt{\left(R_{ \pm}^{0}\right)^{2}-R_{ \pm z}^{2}}
\end{aligned}
$$

which enter the density profiles $H\left(R_{ \pm}\right)$and the flow vectors $u\left(R_{ \pm}\right)$. Since the Lorentz transformation (3.3) mixes the components of $\boldsymbol{r}$, the functions $\eta_{ \pm}(\boldsymbol{r}), \rho_{ \pm}(\boldsymbol{r}), \tau_{ \pm}(\boldsymbol{r})$, and thus the integrand of (3.20) are in general complicated functions of the integration variables $r_{x}, r_{y}$ und $r_{z}$.

The argument $u\left(R_{ \pm}\right) \cdot P_{ \pm}$of the Boltzmann factor can be evaluated in any frame. It turns out to be most convenient to transform $u\left(R_{ \pm}\right)$and $u\left(R_{\mathrm{d}}\right)$ from the f-frame to $u_{ \pm}$and $u_{\mathrm{d}}$ in the d-frame:

$$
u_{ \pm}^{\mu}=L^{\mu \nu} u_{\nu}\left(R_{ \pm}\right), u_{\mathrm{d}}^{\mu}=L^{\mu \nu} u_{\nu}\left(R_{\mathrm{d}}\right) .
$$

Under the assumption that over the effective integration domain the flow and chemical potential can be approximated as constants we find

$$
\begin{aligned}
& \frac{f\left(R_{+}, P_{+}\right) f\left(R_{-}, P_{-}\right)}{f^{2}\left(R_{\mathrm{d}}, \frac{P_{\mathrm{d}}}{2}\right)}= \\
& \quad \exp \left(-\frac{q_{+} \cdot u_{+}+q_{-} \cdot u_{-}-2 m u_{\mathrm{d}}^{0}}{T}\right) \\
& \quad \times \exp \left(-\frac{\rho_{+}^{2}+\rho_{-}^{2}-2 \rho_{\mathrm{d}}^{2}}{2(\Delta \rho)^{2}}-\frac{\eta_{+}^{2}+\eta_{-}^{2}-2 \eta_{\mathrm{d}}^{2}}{2(\Delta \eta)^{2}}\right) .
\end{aligned}
$$

Since in the d-frame the relative momentum $\boldsymbol{q}$ is small, the energy components $q_{ \pm}^{0}$ can be expanded non-relativistically:

$$
\begin{aligned}
q_{+} \cdot u_{+}+q_{-} \cdot u_{-}= & m\left(u_{+}^{0}+u_{-}^{0}\right)+\frac{\boldsymbol{q}^{2}}{2 m}\left(u_{+}^{0} \pm u_{-}^{0}\right) \\
& -\boldsymbol{q} \cdot\left(\boldsymbol{u}_{+}-\boldsymbol{u}_{-}\right)+\frac{B}{2}\left(u_{-}^{0} \mp u_{-}^{0}\right),
\end{aligned}
$$

where $B=m_{\mathrm{d}}-2 m$. The two signs refer to two different treatments of energy conservation: the lower sign applies if one of the two nucleons (here: $q_{-}$) is off-shell as prescribed by Eq. (3.18), the upper sign refers to the case where we simply neglect energy conservation and take both nucleons on-shell as in Eq. (3.1) (in this case the term $\sim B$ vanishes). With harmonic oscillator wave functions the Wigner density of the deuteron is a Gaussian, $\mathcal{D}(\boldsymbol{r}, \boldsymbol{q})=8 \exp \left(-\boldsymbol{r}^{2} / d^{2}-\boldsymbol{q}^{2} d^{2}\right)$, and the integration over $d^{3} q$ in $(3.20)$ is straightforward:

$$
\begin{aligned}
\mathcal{C}_{\mathrm{d}}\left(R_{\mathrm{d}}, P_{\mathrm{d}}\right)= & \int \frac{d^{3} r}{\left(\pi d_{\text {eff }}^{2}\right)^{\frac{3}{2}}} \exp \left(-\frac{\rho_{+}^{2}+\rho_{-}^{2}-2 \rho_{\mathrm{d}}^{2}}{2(\Delta \rho)^{2}}\right) \\
& \times \exp \left(-\frac{\eta_{+}^{2}+\eta_{-}^{2}-2 \eta_{\mathrm{d}}^{2}}{2(\Delta \eta)^{2}}\right) \\
& \times \exp \left(-\frac{\boldsymbol{r}^{2}}{d^{2}}-\frac{m}{T}\left(u_{+}^{0}+u_{-}^{0}-2 u_{\mathrm{d}}^{0}\right)\right) \\
& \times \exp \left(-\frac{B_{\text {eff }}}{T}+\frac{\left(\boldsymbol{u}_{+}-\boldsymbol{u}_{-}\right)^{2}}{4 T^{2} d_{\text {eff }}^{2}}\right) .
\end{aligned}
$$

Here the two parameters $d_{\text {eff }}^{2}=d^{2}+\left(u_{+}^{0} \pm u_{-}^{0}\right) /(2 m T)$ and $B_{\text {eff }}=B\left(u_{-}^{0} \mp u_{-}^{0}\right) / 2$ depend again on how we deal with energy conservation.

\section{B. Integration over relative coordinates}

When trying to perform the integration over $d^{3} r$ one encounters subtle causality problems which require some discussion. The integration in (3.19) runs over the freezeout hypersurface $\tau_{\mathrm{d}}=\tau_{0}$. The hypersurface spanned by the $d^{3} r$ integration at constant time $t_{\mathrm{d}}$ in the d-frame is given in the f-frame by

$$
R^{0}=R_{\mathrm{d}}^{0}+\left(\boldsymbol{R}-\boldsymbol{R}_{\mathrm{d}}\right) \cdot \boldsymbol{b} / b^{0},
$$

which follows by substituting $\boldsymbol{b} \cdot \boldsymbol{R}_{ \pm}$into $R_{ \pm}^{0}$ in Eq. (4.1). This hypersurface is inclined relative to the freeze-out hypersurface, and it also cuts the $T=\left|R_{z}\right|$ half-planes which are tangent to the light cone originating from the 
collision point of the two nuclei. Therefore, when integrating over $d^{3} r$ in $(3.20)$ looking for nucleons which may form a deuteron, the coordinates $R_{ \pm}$leave the freeze-out hypersurface and even the light cone which contains all produced particles.

It turns out that this problem is much less severe than it first appears. First, contributions near the longitudinal light cone are suppressed by the fact that on the light cone the longitudinal proper time $\tau=\sqrt{\left(R^{0}\right)^{2}-R_{z}^{2}}$ vanishes, leading to a diverging flow velocity $u$ whose temporal and longitudinal components are given by $R^{0} / \tau$ and $R_{z} / \tau$, respectively, and, correspondingly, to a vanishing Boltzmann factor $\exp (-p \cdot u(x) / T)$. This constrains the effective integration domain in the longitudinal direction to a region well inside the light cone.

In the transverse directions we must constrain the integration domain by hand to the inside of the light cone, since (as discussed in Sec. IID) our source parametrization does not automatically ensure consistency with causality of the produced particles' positions and velocities. Fortunately, this is not a serious interference: both the deuteron Wigner density and the "homogeneity factor" in (3.20) are peaked at small values of $\boldsymbol{r}$. The latter is the more peaked the more strongly the system expands, i.e. the larger the flow velocity gradients are. As we will see, the integration domain over $\boldsymbol{r}$ is, to the extent that it is not already restricted by the finite range of $\mathcal{D}(\boldsymbol{r}, \boldsymbol{q})$, again given by the homogeneity radii $\mathcal{R}_{i}$ discussed in Sec. IIE. Fortunately, these are smallest for deuterons with large transverse masses where the causality problems are expected to be most serious.

In view of their minor numerical effects, we refrain from giving a detailed account of the technical implementation of these restrictions into the numerical evaluation of (4.6), referring instead to Ref. 45. In practice, the following analytical estimates turn out to be sufficiently accurate even on a quantitative level.

\section{Analytic approximation of the correction factor}

Since the measured deuteron momentum spectra do not contain information on the point of deuteron formation, the relevant quantity is the average correction factor

$$
\left\langle\mathcal{C}_{\mathrm{d}}\right\rangle\left(P_{\mathrm{d}}\right)=\frac{\int P_{\mathrm{d}} \cdot d^{3} \sigma\left(R_{\mathrm{d}}\right) f^{2}\left(R_{\mathrm{d}}, \frac{P_{\mathrm{d}}}{2}\right) \mathcal{C}_{\mathrm{d}}\left(R_{\mathrm{d}}, P_{\mathrm{d}}\right)}{\int P_{\mathrm{d}} \cdot d^{3} \sigma\left(R_{\mathrm{d}}\right) f^{2}\left(R_{\mathrm{d}}, \frac{P_{\mathrm{d}}}{2}\right)} .
$$

We will first calculate $\mathcal{C}_{\mathrm{d}}\left(R_{\mathrm{d}}, P_{\mathrm{d}}\right)$ in analytic approximation for a special combination of $R_{\mathrm{d}}$ and $P_{\mathrm{d}}$ (given by (4.9) below) and then argue that the result, called $\mathcal{C}_{\mathrm{d}}^{0}$, is actually a very good approximation of $\left\langle\mathcal{C}_{\mathrm{d}}\right\rangle\left(P_{\mathrm{d}}\right)$. Numerical studies 45] confirm the validity of these arguments.

We first concentrate on deuterons with zero transverse momentum which are at rest in the fluid cell where they are created, i.e. whose four-velocity $b^{\mu}$ agrees with the flow four-velocity at the production point, $b=u\left(R_{\mathrm{d}}\right)$ :

$$
b^{\mu}=\frac{1}{\tau_{\mathrm{d}}}\left(R_{\mathrm{d}}^{0}, 0,0, R_{\mathrm{d} z}\right) .
$$

In the d-frame the fireball near $R_{\mathrm{d}}$ can then be described non-relativistically as long as the longitudinal flow velocity gradients are sufficiently small. As long as $d<\tau_{\mathrm{d}}$ the non-relativistic approximation is very good in the relevant region $r_{z} \lesssim d$ where $\mathcal{D}$ is non-zero. Since $b_{x}=b_{y}=0$ the Lorentz transformations $(3.3,4.3$ do not mix longitudinal and transverse directions, and the integrand for the coalescence factor has the same axial symmetry as the fireball. Then

$$
\begin{aligned}
& \eta_{ \pm} \approx \eta_{\mathrm{d}} \pm \frac{r_{z}}{2 \tau_{\mathrm{d}}} \\
& \rho_{ \pm}^{2}=\rho_{\mathrm{d}}^{2}+\frac{r_{x}^{2}+r_{y}^{2}}{4} \pm\left(r_{x} R_{\mathrm{d} x}+r_{y} R_{\mathrm{d} y}\right) \\
& \tau_{ \pm} \approx \tau_{\mathrm{d}}-\frac{r_{z}^{2}}{8 \tau_{\mathrm{d}}}
\end{aligned}
$$

after a non-relativistic expansion of (4.2). Further, the flow $u_{ \pm}$follows from $u\left(R_{ \pm}\right)$by a simple shift in the longitudinal rapidity:

$$
\begin{aligned}
& L^{\mu \nu} u_{\nu}(R) \approx \\
& \quad\left(1+\frac{\left(\eta-Y_{\mathrm{d}}\right)^{2}}{2}+\frac{\eta_{f}^{2} \rho^{2}}{2(\Delta \rho)^{2}}, \frac{\eta_{f} R_{x}}{\Delta \rho}, \frac{\eta_{f} R_{y}}{\Delta \rho}, \eta-Y_{\mathrm{d}}\right),
\end{aligned}
$$

where we have already used the saddle-point approximation (2.13). For the given values of $d$ and $m T$ the value of

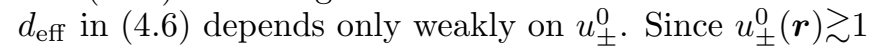
for $b=u\left(R_{\mathrm{d}}\right)$ and small $|\boldsymbol{r}|$, we use $d_{\text {eff }}^{2} \approx d^{2}+1 / m T$ and $B_{\text {eff }}=0$, or $d_{\text {eff }}^{2} \approx d^{2}$ and $B_{\text {eff }} \approx B$, respectively, depending on whether or not energy conservation is taken into account. With these approximations 4.6 turns into a product of Gaussian integrals in $r_{x}, r_{y}$ and $r_{z}$, with the result

$$
\begin{aligned}
\mathcal{C}_{\mathrm{d}}^{0} & =\frac{1}{\gamma_{\perp}^{2} \gamma_{\|}}\left(\frac{d}{d_{\mathrm{eff}}}\right)^{3} \exp \left(\frac{B_{\mathrm{eff}}}{T}\right) \\
\gamma_{\perp} & =\sqrt{1+\left(\frac{d}{2 \mathcal{R}_{\perp}(m)}\right)^{2}-\left(\frac{\eta_{f}}{2 T \Delta \rho}\right)^{2}} \\
\gamma_{\|} & =\sqrt{1+\left(\frac{d}{2 \mathcal{R}_{\|}(m)}\right)^{2}-\left(\frac{1}{2 T \tau_{\mathrm{d}}}\right)^{2}} .
\end{aligned}
$$

For the source parameters given in Sec. IIF the last terms under the square root in $\gamma_{\perp}, \gamma_{\|}$are negligible. They originate from the coupling term $\boldsymbol{q} \cdot\left(\boldsymbol{u}_{+}-\boldsymbol{u}_{-}\right)$in (4.5) and the resulting term $\left|\boldsymbol{u}_{+}-\boldsymbol{u}_{-}\right|^{2}$ in (4.6). They would thus be absent if we had started from the approximation (3.21) instead of (3.20). The smallness of these terms is a good check of the accuracy of (3.21).

The last two factors in (4.11a) deviate from unity by less than $2 \%$ for temperatures $T$ between $100 \mathrm{MeV}$ and 
$170 \mathrm{MeV}$ if energy conservation is properly accounted for; if not, the deviations have the opposite sign, but remain below $5 \%$.

Given the high accuracy of (3.21), we can use it for a check of the sensitivity of $\mathcal{C}_{\mathrm{d}}^{0}$ on the choice of the internal deuteron wave function. A numerical integration of (3.21) with the Hulthen wave function (3.4a) yields values for $\mathcal{C}_{\mathrm{d}}^{0}$ which are somewhat larger than those for harmonic oscillator wave functions. For the source parameters given in Sec. IIF we obtain for the harmonic oscillator wave function $\mathcal{C}_{\mathrm{d}}^{0}=0.81_{-0.05}^{+0.03}$ and for the Hulthen form $\mathcal{C}_{\mathrm{d}}^{0}=0.84_{-0.04}^{+0.02}$ (where the upper and lower limits indicate the effects from the estimated uncertainties in $\left.\left(T, \eta_{f}\right)\right)$. The numbers in Table 1 show that the differences are sensitive mainly to the transverse and longitudinal flow gradients; they remain on the level of a few percent for weakly expanding sources (leftmost column), become stronger for more rapidly expanding sources (rightmost column), and can reach a factor of 2 or 3 in systems with very small interaction volume ( $p p$ collisions).

The origin of the difference is readily understood: while both wave functions provide the same rms radius, the maximum of $r^{2}\left|\varphi_{\mathrm{d}}(r)\right|^{2}$ is at $r \approx 1.5 \mathrm{fm}$ for the Hulthen form and at $r \approx 3 \mathrm{fm}$ for the harmonic oscillator. Since the "homogeneity factor" in (3.21) peaks at small values of $r$, especially for strongly expanding systems with small homogeneity radii, the integral is larger for the more realistic Hulthen wave function than for the harmonic oscillator one.

Numerical calculations show that $\mathcal{C}_{\mathrm{d}}\left(R_{\mathrm{d}}, P_{\mathrm{d}}\right)$ varies much less as a function of $R_{\mathrm{d}}$ than $f^{2}\left(R_{\mathrm{d}}, P_{\mathrm{d}} / 2\right)$. On the other hand, the particular point $\bar{R}_{\mathrm{d}}$ with $u\left(\bar{R}_{\mathrm{d}}\right)=b$ at which $\mathcal{C}_{\mathrm{d}}^{0}$ was evaluated corresponds to the maximum of $\mathcal{C}_{\mathrm{d}}\left(R_{\mathrm{d}}, P_{\mathrm{d}}\right)$, to the maximum of the Boltzmann part of $f\left(R_{\mathrm{d}}, P_{\mathrm{d}} / 2\right)$, and thus approximately to the maximum of integrand in the numerator of (4.8). We can therefore pull $\mathcal{C}_{\mathrm{d}}\left(\bar{R}_{\mathrm{d}}, P_{\mathrm{d}}\right)=\mathcal{C}_{\mathrm{d}}^{0}$ in front of that integral and thus have $\left\langle\mathcal{C}_{\mathrm{d}}\right\rangle \approx \mathcal{C}_{\mathrm{d}}^{0}$. A numerical check gave $\left\langle\mathcal{C}_{\mathrm{d}}\right\rangle\left(\boldsymbol{P}_{\mathrm{d}}=\mathbf{0}\right)=0.79$ instead of $\mathcal{C}_{\mathrm{d}}^{0}=0.81$.

For a boost-invariant source, where every comoving observer has identical surroundings, we expect $\mathcal{C}_{\mathrm{d}}\left(R_{\mathrm{d}}, P_{\mathrm{d}}\right)$ to depend only on the difference between the local flow velocity and the deuteron's velocity, $\mathcal{C}_{\mathrm{d}}\left(R_{\mathrm{d}}, P_{\mathrm{d}}\right)=$ $\mathcal{C}_{\mathrm{d}}\left(u\left(R_{\mathrm{d}}\right)-b\right)$, and $\left\langle\mathcal{C}_{\mathrm{d}}\right\rangle$ to be independent of $P_{\mathrm{d}}$. In our fireball longitudinal boost-invariance is broken by the density profile $H(R)$. However, for the Gaussian profile used here $\left\langle\mathcal{C}_{\mathrm{d}}\right\rangle$ still turns out to be independent of the deuteron's longitudinal rapidity. In the transverse direction we have no boost-invariance at all. As a consequence we find a slight decrease of $\left\langle\mathcal{C}_{\mathrm{d}}\right\rangle$ with increasing transverse velocity of the deuteron. Deuterons with non-zero transverse velocity see the fireball Lorentz-contracted in their direction of motion; this decreases the corresponding length of homogeneity and thus $\left\langle\mathcal{C}_{\mathrm{d}}\right\rangle$. In the region $m_{t} / m \leq 1.25$, i.e. $v_{t} \leq 0.6 c$, to which we restrict our discussion in the case of Gaussian transverse density profiles (see the discussion at the end of Sec. IID), this effect is small and $\left\langle\mathcal{C}_{\mathrm{d}}\right\rangle$ is approximately constant.

We can summarize the results of this section in the following approximate formula for the quantum mechanical correction factor in terms of the deuteron size parameter $d$ and the longitudinal and transverse lengths of homogeneity for nucleons:

$$
\left\langle\mathcal{C}_{\mathrm{d}}\right\rangle \approx \frac{1}{\left(1+\left(\frac{d}{2 \mathcal{R}_{\perp}(m)}\right)^{2}\right) \sqrt{1+\left(\frac{d}{2 \mathcal{R}_{\|}(m)}\right)^{2}}}
$$

This expression does not depend on the longitudinal rapidity and, for small transverse velocities, only weakly on the transverse momentum of the deuteron. Applying this expression to $\mathrm{p}+\mathrm{p}$ collisions and inserting correspondingly for the homogeneity lengths about $1 \mathrm{fm}$ each one obtains $\left\langle\mathcal{C}_{\mathrm{d}}\right\rangle \approx 0.15$ in good numerical agreement with Hagedorn's value (3.22).

\section{LARGER CLUSTERS: ${ }_{1}^{3} \mathrm{H}$ AND ${ }_{2}^{3} \mathrm{HE}$}

In this section we give a quick estimate of the quantum mechanical correction factor for clusters made of three nucleons, i.e. for ${ }^{3} \mathrm{H}$ and ${ }^{3} \mathrm{He}$. Clearly, for three nucleons the internal wave function and its Wigner transform is much more complicated than for deuterons. For the three nucleons with coordinates $\boldsymbol{r}_{1}, \boldsymbol{r}_{2}$ and $\boldsymbol{r}_{3}$, we introduce the c.m. coordinates $\boldsymbol{R}=\left(\boldsymbol{r}_{1}+\boldsymbol{r}_{2}+\boldsymbol{r}_{3}\right) / 3$ and the relative coordinates $\boldsymbol{\rho}=\left(\boldsymbol{r}_{1}-\boldsymbol{r}_{2}\right) / \sqrt{2}$ and $\boldsymbol{\lambda}=\left(\boldsymbol{r}_{1}+\boldsymbol{r}_{2}-2 \boldsymbol{r}_{3}\right) / \sqrt{6}$. With this choice we have $\boldsymbol{r}_{1}^{2}+\boldsymbol{r}_{2}^{2}+\boldsymbol{r}_{3}^{2}=\boldsymbol{R}^{2}+\boldsymbol{\rho}^{2}+\boldsymbol{\lambda}^{2}$ and $d^{3} r_{1} d^{3} r_{2} d^{3} r_{3}=3^{\frac{3}{2}} d^{3} R d^{3} \rho d^{3} \lambda$. As before we approximate the internal wave function by a spherical harmonic oscillator solution 65:

$$
\varphi\left(\boldsymbol{r}_{1}, \boldsymbol{r}_{2}, \boldsymbol{r}_{3}\right)=\left(3 \pi^{2} b^{4}\right)^{-3 / 4} \exp \left(-\frac{\boldsymbol{\rho}^{2}+\boldsymbol{\lambda}^{2}}{2 b^{2}}\right) .
$$

This wave function is normalized and has the rms radius b:

$$
\begin{aligned}
& \int 3^{3 / 2} d^{3} \rho d^{3} \lambda\left|\varphi\left(\boldsymbol{r}_{1}, \boldsymbol{r}_{2}, \boldsymbol{r}_{3}\right)\right|^{2}=1 \\
& r_{\mathrm{rms}}^{2}=\int 3^{3 / 2} d^{3} \rho d^{3} \lambda \frac{\rho^{2}+\lambda^{2}}{3}\left|\varphi\left(\boldsymbol{r}_{1}, \boldsymbol{r}_{2}, \boldsymbol{r}_{3}\right)\right|^{2}=b^{2}
\end{aligned}
$$

Both clusters are spin- $\frac{1}{2}$ fermions, and the binding energies and rms radii are approximately $-8 \mathrm{MeV}$ and $1.75 \mathrm{fm}$. (Note that this rms radius is smaller than for deuterons!) ${ }^{3} \mathrm{He}$ is somewhat more loosely bound than ${ }^{3} \mathrm{H}$, but we neglect this difference here.

To estimate $\mathcal{C}_{\mathrm{t}}^{0}$ for $\mathrm{a}^{3} \mathrm{H} /{ }^{3} \mathrm{He}$ cluster at rest in the center of the fireball, with momentum $P=(3 m, \boldsymbol{P}=\mathbf{0})$ and c.m. coordinates $R=\left(R^{0}, \boldsymbol{R}=\mathbf{0}\right)$, we evaluated numerically 


$$
\begin{aligned}
\mathcal{C}_{\mathrm{t}}^{0} \approx & \int 3^{\frac{3}{2}} d^{3} \rho d^{3} \lambda\left|\varphi\left(\boldsymbol{r}_{1}, \boldsymbol{r}_{2}, \boldsymbol{r}_{3}\right)\right|^{2} \\
& \times \frac{f\left(R_{1}, \frac{P}{3}\right) f\left(R_{2}, \frac{P}{3}\right) f\left(R_{3}, \frac{P}{3}\right)}{f^{3}\left(R, \frac{P}{3}\right)}
\end{aligned}
$$

where $R_{i}=\left(R^{0}, \boldsymbol{r}_{i}\right)$ are the space-time coordinates of the three nucleons. The results $\mathcal{C}_{\mathrm{t}}^{0}=0.78_{-0.06}^{+0.05}$ for $\tau_{0}=9 \mathrm{fm} / c$ and $\mathcal{C}_{\mathrm{t}}^{0}=0.67_{-0.07}^{+0.06}$ for $\tau_{0}=6 \mathrm{fm} / c$, respectively, are not much smaller than the corresponding values for the deuteron. Whereas one would generically expect larger flow effects for three than for two nucleons, the three nucleons in the ${ }^{3} \mathrm{H} /{ }^{3} \mathrm{He}$ clusters occupy a smaller and therefore more homogeneous region around their center of mass.

As for the deuteron we expect that $\mathcal{C}_{\mathrm{t}}^{0}$ provides a good estimate for the average correction factor $\left\langle\mathcal{C}_{t}\right\rangle$. A more rigorous calculation must, however, take into account the binding energy which is larger and thus more important than that of the deuteron. Unlike deuterons, tritons and ${ }^{3} \mathrm{He}$ can be formed via an excited state by coalescence of three nucleons without requiring additional particles for energy-momentum conservation.

\section{EXTRACTING PHYSICS FROM MEASURED CLUSTER SPECTRA}

\section{A. The invariant coalescence factor $B_{A}$}

According to (3.19), the invariant cluster spectra are given by (2.14) multiplied by the quantum mechanical correction factor $\left\langle\mathcal{C}_{A}\right\rangle(P)$ :

$$
\begin{aligned}
E \frac{d N_{A}}{d^{3} P} \approx & M_{t} \frac{2 J_{A}+1}{(2 \pi)^{3}} e^{\frac{\mu_{A}-M}{T}}\left\langle\mathcal{C}_{A}\right\rangle(P) V_{\mathrm{eff}}\left(A, M_{t}\right) \\
& \times \exp \left(-\frac{M_{t}-M}{T^{*}}-\frac{A Y^{2}}{2(\Delta \eta)^{2}}\right) .
\end{aligned}
$$

For the invariant coalescence factor $B_{A}$ defined by (1.1) we thus find

$$
B_{A}=\frac{2 J_{A}+1}{2^{A}} A\left\langle\mathcal{C}_{A}\right\rangle \frac{V_{\mathrm{eff}}\left(A, M_{t}\right)}{V_{\mathrm{eff}}\left(1, m_{t}\right)}\left(\frac{(2 \pi)^{3}}{m_{t} V_{\mathrm{eff}}\left(1, m_{t}\right)}\right)^{A-1} .
$$

The factor $A$ arises from $M_{t} / m_{t}^{A}=A / m_{t}^{A-1}$. (In case of a static, non-expanding fireball the homogeneity volume $V_{\text {eff }}$ in this expression would be replaced by the total fireball volume $V_{\text {cov }}$ 15.) With $V_{\text {eff }}$ given by Eqs. (2.18,2.19), we can write $B_{2}$ as

$$
B_{2}=\frac{3 \pi^{3 / 2}\left\langle\mathcal{C}_{\mathrm{d}}\right\rangle}{2 m_{t} \mathcal{R}_{\perp}^{2}\left(m_{t}\right) \mathcal{R}_{\|}\left(m_{t}\right)} .
$$

Note that the last exponential factor in (6.1), which depends strongly on $M_{t}$ and $Y$, has cancelled in the ratio. With $\left\langle\mathcal{C}_{\mathrm{d}}\right\rangle$ given by (4.12), $B_{2}$ can thus be expressed completely in terms of the deuteron size $d$ and the homogeneity lengths ("HBT radii") $\mathcal{R}_{\perp}, \mathcal{R}_{\|}$.

Eq. (6.3) implies that, in the model of Sec. II, $B_{A}$ is almost momentum independent: both $m_{t} V_{\text {eff }}\left(1, m_{t}\right)$ and $\left\langle\mathcal{C}_{A}\right\rangle$ depend only very weakly on $m_{t}$ and $Y$. An important precondition for this weak $m_{t}$-dependence of $B_{2}$ is, of course, the cancellation of the $\exp \left(-M_{t} / T^{*}\right)$ factors; as discussed in Sec. IID, the latter is due to the Gaussian form of the transverse density profile in $H(R)$ which according to (2.16) causes identical inverse slope parameters $T^{*}$ for all clusters. We have mentioned before that this is inconsistent with the measurements (see Sec. VIIB below), and that more box-like transverse density profiles are phenomenologically preferred. In this case Eq. (6.3) must be amended as follows:

$$
B_{2}=\frac{3 \pi^{3 / 2}\left\langle\mathcal{C}_{\mathrm{d}}\right\rangle}{2 m_{t} \mathcal{R}_{\perp}^{2}\left(m_{t}\right) \mathcal{R}_{\|}\left(m_{t}\right)} e^{2\left(m_{t}-m\right)\left(\frac{1}{T_{\mathrm{P}}^{*}}-\frac{1}{T_{\mathrm{d}}^{*}}\right)} .
$$

Eqs. 4.12,6.4 are the most important theoretical results of the present paper.

Since neutrons are hard to measure, experiments usually do not provide $B_{A}$, but rather

$$
\begin{aligned}
B_{A}^{*} & =E_{A} \frac{d N_{A}}{d^{3} P_{A}} /\left.\left(E_{\mathrm{p}} \frac{d N_{\mathrm{p}}}{d^{3} P_{\mathrm{p}}}\right)^{Z+N}\right|_{P_{\mathrm{p}}=P_{A} / A} \\
& =B_{A} \exp \left(\frac{N\left(\mu_{\mathrm{n}}-\mu_{\mathrm{p}}\right)}{T}\right)
\end{aligned}
$$

Here possibly different chemical potentials for neutrons and protons are important: If nucleons and antinucleons have the same temperature, flow and freeze-out density distribution, as we have assumed, our model yields identical coalescence factors $B_{A}$ and $B_{\bar{A}}$ for clusters made of matter and antimatter. For $\mu_{\mathrm{n}} \neq \mu_{\mathrm{p}}$ the corresponding values $B_{A}^{*}$ and $B_{\bar{A}}^{*}$ will, however, be different, and they will also differ from $B_{A}$. If the initial neutron excess of the cold $\mathrm{Pb}$ nuclei were still present at freeze-out, we would expect for $\mathrm{Pb}+\mathrm{Pb}$ collisions $B_{\mathrm{d}}^{*} \approx 1.5 B_{\mathrm{d}} \approx 2.3 B_{\mathrm{d}}^{*}$, i.e. quite large differences. Of course, it is not likely that the large net isospin remains in the neutron channel until freeze-out; a considerable fraction is expected to boil off with other produced particles. Nevertheless, there may be a visible effect of $\mu_{\mathrm{n}} \neq \mu_{\mathrm{p}}$ on $B_{2}^{*}$ and especially on $B_{3}^{*}$ for ${ }^{3} \mathrm{H}$ (which contains 2 neutrons), as well as a characteristic difference in the $B_{3}^{*}$ values for ${ }^{3} \mathrm{H}$ and ${ }^{3} \mathrm{He}$.

\section{B. Cluster fugacities}

From the ratio of particles to antiparticles the fugacities can be calculated:

$$
\left.\frac{E_{A} \frac{d N_{A}}{d^{3} P_{A}}}{E_{\bar{A}} \frac{d N_{\bar{A}}}{d^{3} P_{\bar{A}}}}\right|_{P_{A}=P_{\bar{A}}}=\exp \left(\frac{2 \mu_{A}}{T}\right)=\lambda_{A}^{2} .
$$


Using the proton fugacity $\lambda_{\mathrm{p}}=\exp \left(\mu_{\mathrm{p}} / T\right)$ from the ratio $\mathrm{p} / \overline{\mathrm{p}}$ and the deuteron fugacity $\lambda_{\mathrm{d}}=\exp \left(\left(\mu_{\mathrm{p}}+\mu_{\mathrm{n}}\right) / T\right)$ from the ratio $\mathrm{d} / \overline{\mathrm{d}}$ one can in principle extract the neutron fugacity $\lambda_{\mathrm{n}}=\exp \left(\mu_{\mathrm{n}} / T\right)$. Once the temperature is known (see below), the separate chemical potentials of neutrons and protons can thus be determined. In practice, however, the uncertainties in the $\mathrm{p}$ and $\overline{\mathrm{p}}$ spectra from insufficiently well known $\Lambda$ and $\bar{\Lambda}$ decay contaminations and the large statistical error bars on the $\overline{\mathrm{d}}$ spectrum limit the usefulness of such an analysis.

\section{Freeze-out temperature from cluster ratios}

The thermal freeze-out temperature for pions was determined from a simultaneous analysis of their spectra and two-particle correlations, see Sec. IIF. The same freeze-out parameters appear to also describe quite well the proton $m_{t}$-spectra [66]. An independent determination of the proton freeze-out temperature uses the chemical composition rather than the shape of the momentum distribution at freeze-out. In particular one can try to analyse the ratio

$$
S_{A A^{\prime}}=\left(E_{A} \frac{d N_{A}}{d^{3} P_{A}}\right) /\left.\left(E_{A^{\prime}} \frac{d N_{A^{\prime}}}{d^{3} P_{A^{\prime}}}\right)\right|_{\boldsymbol{P}_{A}=\boldsymbol{P}_{A^{\prime}}=\mathbf{0}}
$$

of the invariant spectra at zero momentum of two different types of clusters (including nucleons, $A=1$ ). To investigate such a possibility is suggested in particular by the data from the NA52 experiment at CERN 67] and from the E864 experiment at the AGS 68 which measure cluster yields only very close to $P_{t}=0$. With (6.1) this ratio is given by

$$
\begin{aligned}
& S_{A A^{\prime}}=\frac{2 J_{A}+1}{2 J_{A^{\prime}}+1} \lambda_{\mathrm{p}}^{Z_{A}-Z_{A^{\prime}}} \lambda_{\mathrm{n}}^{N_{A}-N_{A^{\prime}}} e^{\left(A^{\prime}-A\right) \frac{m}{T}} \\
& \times \frac{\left\langle\mathcal{C}_{A}\right\rangle}{\left\langle\mathcal{C}_{A^{\prime}}\right\rangle} \frac{A V_{\text {eff }}\left(A, M_{A}\right)}{A^{\prime} V_{\text {eff }}\left(A^{\prime}, M_{A^{\prime}}\right)}
\end{aligned}
$$

which for known fugacities $\lambda_{\mathrm{n}, \mathrm{p}}$ is easily solved for the temperature $T$. [For antiparticles $Z$ and $N$ must be taken negative in (6.8).]

The main error of such a determination of the temperature does not arise from the correction factors $\left\langle\mathcal{C}_{A}\right\rangle$, which do not vary much in our parameter range, but from the sometimes substantial uncertainties of the experimental value of $S_{A A^{\prime}}$ and of the fugacities. Furthermore, the temperature value determined from the yield ratio at a certain point in momentum space rather than from the ratio of total yields depends on model assumptions about the shape of the spectra. For example, we already mentioned that a box profile for the transverse density distribution gives better results for the slope systematics of the transverse cluster spectra than the Gaussian profile from which (6.1) was derived. (This change does not affect the rapidity spectra.) Adjusting the box radius such that the same rms radius $\Delta \rho$ is reproduced $\left(\rho_{\text {box }}=2 \Delta \rho\right)$, we find instead of 6.8$)$ the same expression multiplied by a factor $\kappa_{A} / \kappa_{A^{\prime}}$ where

$$
\kappa_{A}=\left(1-e^{-\frac{2 M_{A} \eta_{f}^{2}}{T}}\right)\left(1+\frac{T}{m \eta_{f}^{2}}\right) .
$$

(We used the same saddle-point approximation as in the derivation of (6.1), see Sec. [I. We also kept the strength of the flow $\eta_{f} / \Delta \rho$ fixed when switching from the Gaussian to the box profile.) For sufficiently large transverse flow $\eta_{f}^{2} \gg T / m$ this $\kappa_{A}$ is just a factor 1 without any consequences. In the opposite limit, however, $\lim _{\eta_{f} \rightarrow 0} \kappa_{A}=2 A$, i.e. the intercept of the invariant spectrum at $P_{t}=0$ is changed by a factor $2 A$. Similarly, changing the Gaussian longitudinal density distribution into a box doesn't matter much for systems with strong longitudinal expansion, but yields another factor $\sqrt{A}$ for static systems. For static systems this just compensates the factor $A^{-3 / 2}$ from Eq. (2.19), which is intuitively correct since for static fireballs with constant density the effective volume $V_{\text {eff }}$ must coincide with the total fireball volume, independent of $A$.

Parametrizing the last factors in $S_{A A^{\prime}}$ as

$$
\frac{\kappa_{A}}{\kappa_{A^{\prime}}} \frac{A V_{\mathrm{eff}}\left(A, M_{A}\right)}{A^{\prime} V_{\mathrm{eff}}\left(A^{\prime}, M_{A^{\prime}}\right)}=\left(\frac{A}{A^{\prime}}\right)^{\chi},
$$

we thus find find $-\frac{1}{2} \lesssim \chi \leq 1$, with the upper limit reproducing the behaviour for a static, homogeneous fireball and the lower limit corresponding to rapidly expanding systems or systems with a Gaussian transverse density profile. For the source parameters given in Sec. IIF we expect to be close to $\chi=-\frac{1}{2}$. However, in order to allow for this kind of model-dependence, freeze-out temperatures extracted from $S_{A A^{\prime}}$ should be plotted against the power $\chi$.

\section{COMPARISON WITH EXPERIMENT}

In this section we discuss the presently available cluster data from $\mathrm{Pb}+\mathrm{Pb}$ collisions at the CERN-SPS, taken by the NA52 and NA44 collaborations. The NA52 experiment does not trigger on collision centrality and measures particles close to $P_{t}=0$, with different rigidities $P / Z$ of the spectrometer magnets providing data at various rapidities. Recently, the minimum bias data were re-analyzed to extract the centrality of the collisions. Although the minimum bias data contain information on $\mathrm{p}, \mathrm{d},{ }^{3} \mathrm{H},{ }^{3} \mathrm{He}, \overline{\mathrm{p}}, \overline{\mathrm{d}}$ and ${ }^{3} \mathrm{He}$ 67, their analysis in the framework of the present model is difficult: the assumed axial symmetry of the source applies only to central collisions, and also the model parameters given in Sec. IIF were extracted from very central $\mathrm{Pb}+\mathrm{Pb}$ collisions. For this reason only the impact parameter selected data from the $4 \%$ most central $\mathrm{Pb}+\mathrm{Pb}$ collisions will be really useful for us. At the Quark Matter '97 conference preliminary $\Lambda, \bar{\Lambda}$-corrected particle ratios $\overline{\mathrm{p}} / \mathrm{p}, \overline{\mathrm{d}} / \mathrm{d}, B_{2}\left(\mathrm{~d} / \mathrm{p}^{2}\right)$ 
and $B_{2}\left(\overline{\mathrm{d}} / \overline{\mathrm{p}}^{2}\right)$ were presented for $y=3.75$ as a function of centrality [4]. They were recently amended by a correction to the proton spectra from $\Delta$-decays $[69]$.

From NA44 we have the $M_{t}$ spectra of $\mathrm{p}, \mathrm{d}$ and $B_{2}\left(\mathrm{~d} / \mathrm{p}^{2}\right)$ [7, 8 as well as an $M_{t}$-integrated ratio $\overline{\mathrm{d}} / \mathrm{d} \approx$ $(0.4-1.5) \cdot 10^{-3}$ [70 in the rapidity range $1.9 \leq y \leq 2.3$ for the $20 \%$ most central collisions.

Finally, we have data for $B_{2}$ and $B_{3}$ from $\mathrm{Au}+\mathrm{Au}$ collisions at the AGS with similar centrality $\left(4 \% \sigma_{\text {tot }}\right)$, but for the considerably lower beam energy of $11.5 \mathrm{GeV} /$ nucleon [32]. Comparison with these data requires a readjustment of the source parameters.

A full comparison between theory and data is possible only for the cluster spectra taken in very central (about $\left.4 \% \sigma_{\text {tot }}\right) \mathrm{Pb}+\mathrm{Pb}$ collisions, since the model parameter set of Sec. IIF describes this class of collisions only. The one data point at this centrality provided by NA52 can therefore only be used to test the absolute normalization of (6.1) or the value (6.2) of $B_{A}$ and does not give information on the spectral behavior. On the other hand, the $M_{t}$-shape of $(6.1)$ can be tested qualitatively with the NA44 data although, due to the weaker centrality cut, the normalization and the slope parameters may be somewhat different from what we expect on the basis of the parameters from Sec. IIF.

\section{A. NA52 data}

From the parameters given in section $\mathrm{IIF}$ and from our values $\left\langle\mathcal{C}_{\mathrm{d}}\right\rangle \approx 0.8$ and $\left\langle\mathcal{C}_{\mathrm{t}}\right\rangle \approx\left\langle\mathcal{C}_{3_{\mathrm{He}}}\right\rangle \approx 0.7$ for the quantum mechanical correction factors, we estimate the invariant coalescence factors $B_{A}$ in $4 \% \sigma_{\text {tot }} \mathrm{Pb}+\mathrm{Pb}$ collisions by using (6.2):

$$
\begin{aligned}
& B_{2} \approx\left(6_{+4}^{-2}\right) \cdot 10^{-4} \mathrm{GeV}^{2}, \\
& B_{3} \approx\left(2_{+4}^{-1}\right) \cdot 10^{-7} \mathrm{GeV}^{4} .
\end{aligned}
$$

Fig. 1 shows the preliminary NA52 data [4] for $B_{2}$ as a function of collision centrality, with the most central collisions on the left. The agreement with our estimate is very good, both for $\mathrm{d}$ and $\overline{\mathrm{d}}$, but we should note that it deteriorates somewhat if the recently reported $\Delta$ corrected preliminary data 69] are used for the comparison. A small neutron excess at nucleon freeze-out may explain the sytematically slightly lower $B_{2}$ values for antideuterons (see Eq. (6.5)). Fig. 1 shows an increase of $B_{2}$ by up to a factor 8 for more peripheral collisions, in qualitative agreement with the naive expectation of decreasing homogeneity lengths and a smaller effective volume in less central collisions.

From the preliminary $\overline{\mathrm{p}} / \mathrm{p}$ and $\overline{\mathrm{d}} / \mathrm{d}$ ratios at $y=3.75$ [4] and a preliminary $\mathrm{d} / \mathrm{p}$ ratio [6] in the most central $\left(4 \% \sigma_{\text {tot }}\right)$ impact parameter bin we tried to determine the freeze-out temperature using (6.8). If we assume a common nucleon fugacity corresponding to $\mu / T=1.5$, we obtain $T \approx(144 \pm 5) \mathrm{MeV}$ for Gaussian density profiles $(\chi=-0.5)$ and $T \approx(124 \pm 4) \mathrm{MeV}$ for a weakly expanding homogeneous fireball $\left(\chi=1\right.$, but still with $\left.\left\langle\mathcal{C}_{\mathrm{d}}\right\rangle=0.8\right)$. For a larger nucleon chemical potential of $\mu / T=1.9$ we find $T \approx(135 \pm 5) \mathrm{MeV}$ and $T \approx(118 \pm 4) \mathrm{MeV}$, respectively. The given range of $\mu / T$ at thermal nucleon freezeout and the resulting uncertainties for the temperature are due to the experimental errors on the particle ratios. Please note that the different values for $\chi$ cause a difference of almost $20 \mathrm{MeV}$ in the extracted temperature. For a purely thermal model with $\chi=1$ and $\left\langle\mathcal{C}_{\mathrm{d}}\right\rangle=1$, NA52 give a freeze-out temperature of $T \approx(115 \pm 10) \mathrm{MeV}$ 沺, taking all uncertainties into account. For a transverse box and longitudinal Gaussian density profile (as will be motivated below), we have $\chi \approx 0.5$, and using again $\left\langle\mathcal{C}_{\mathrm{d}}\right\rangle=0.8$ we obtain $T \approx(125 \pm 10) \mathrm{MeV}$. Given the systematic uncertainties, all these values are consistent with the model parameters given in Sec. IIF.

\section{B. NA44 data}

In Fig. 2 we show the $M_{t}$ distributions of protons, deuterons, and $B_{2}$ from our model. These figures grew out of an analysis of preliminary data by the NA44 collaboration which were made available to us by M. Murray [8], but have so far not been published (which is the reason why no data are shown). A fit of the $M_{t}$-dependence of the invariant proton and deuteron momentum spectra with the function $\exp \left(-\left(M_{t}-M\right) / T^{*}\right)$ yielded effective temperatures $T_{\mathrm{p}}^{*} \approx 250 \mathrm{MeV}$ and $T_{\mathrm{d}}^{*} \approx 350 \mathrm{MeV}$, respectively, i.e. a considerably larger value for deuterons than for protons. As a consequence, the measured $B_{2}$ rises with $M_{t}$ by about a factor $2-3$ in the transverse mass region $m_{t} / m=1.00-1.25$. Higher values of $T^{*}$ for deuterons than for protons have been repeatedly observed 52 54 and are in clear contradiction with the prediction of identical slope parameters (2.16) from a Gaussian source model.

Recently, several possibilities were suggested to account for this discrepancy [55]: a transverse flow profile (2.7) with $\alpha=0.5$, a box profile for the transverse density distribution, and surface emission of clusters. We have analyzed the first two possibilities.

Starting from (2.12) with a linear transverse flow profile $\alpha=1$ and the source parameters of Sec. IIF, we first selected different combinations of $\left(T, \eta_{f}, \frac{\mu}{T}\right)$ which all gave good fits to the proton spectrum (Fig. 2a). The lines for the deuteron and $B_{2}$ spectra in Figs. 2 b b c follow then self-consistently from the parameters used in the proton spectrum without further adjustment. Line no. 6 in Figs. 2f b,c corresponds to the parameter triplet (140 $\mathrm{MeV}, 0.34,1.75)$; it clearly fails to reproduce the deuteron and $B_{2}$ spectra. Combinations with larger temperatures and lower transverse flow rapidities and vice versa lead to deuteron spectra with the same slope, but somewhat different normalizations. The problem of different $\mathrm{p}$ and $\mathrm{d}$ slopes cannot be resolved in this way. Please note 
that there is only a small temperature window for which the fugacity $\mu / T$ needed to reproduce the normalization of the proton spectrum compares well with the value $1.5 \lesssim \mu / T \lesssim 2.0$ from the experimental $\overline{\mathrm{d}} / \mathrm{d}$ ratio; due to the high nucleon mass, the nucleon and cluster yields are highly sensitive to the temperature. The fits tend to under- rather than overestimate the deuteron yields; this eliminates the simple suggestion that deuterons from the center of the fireball (which tend to have smaller $P_{t}$ ) are absorbed by rescattering, leaving only a thin surface shell contributing to the observed deuteron yield, because this would further aggravate the normalization problem.

In a second step, we tested for fixed $T=140 \mathrm{MeV}$ and $\mu / T=1.75$ different transverse flow profiles (2.7). For $\alpha=0.5$ and $\alpha=2$ good fits for the proton spectrum can be obtained with $\eta_{f}=0.38$ and $\eta_{f}=0.24$, respectively. The effects of the modified flow profile on the deuteron and $B_{2}$ spectra are small: lines no. 5 correspond to $\alpha=$ 0.5 , which results in a weak, but insufficient rise of $B_{2}$ with $M_{t} ; \alpha=2$ (lines no. 7) made things worse.

Finally, we fixed $\mu / T=1.75$ and tested transverse box profiles with different radii $\rho_{\text {box }}$ for the function $H(R)$ at different lifetimes $\tau_{0}$. As before, the remaining parameters $T$ and $\eta_{f}$ are adjusted to provide good fits to the proton spectrum. Lines no. 1 to no. 4 correspond to the following combinations of $\left(\tau_{0}, \rho_{\mathrm{box}}, T, \eta_{f}, \mu / T\right)$, respectively (values of $\eta_{f}$ refer to (2.7) with fixed $\Delta \rho=7 \mathrm{fm}$ !):

1. $(7 \mathrm{fm} / c, 10 \mathrm{fm}, 142 \mathrm{MeV}, 0.40,1.75)$;

2. (7 fm/c, $12 \mathrm{fm}, 136 \mathrm{MeV}, 0.34,1.75)$;

3. ( $8 \mathrm{fm} / c, 12 \mathrm{fm}, 134 \mathrm{MeV}, 0.36,1.75)$; and

4. $(9 \mathrm{fm} / c, 14 \mathrm{fm}, 126 \mathrm{MeV}, 0.28,1.75)$.

All four data sets reproduce the slope of the preliminary deuteron and $B_{2}$ data very nicely. They differ somewhat in the normalization of the deuteron spectrum. The preliminary data are located between curves 1 and 3, close to curve 2. The parameter choice no. 4 seems to be the most appropriate with respect to the Gaussian parameter set of Sec. IIF, since $\rho_{\text {box }}=2(\Delta \rho) \approx 14 \mathrm{fm}$ for the box radius leads to the same transverse rms radius of the source (which is the observable determined from pion interferometry) as the Gaussian profile. However, it underestimates the yield of the deuterons and the value of $B_{2}$, indicating too large an effective source volume for nucleons (see Sec. IIE and Eq. 6.2). Better results are obtained with somewhat smaller values of $\rho_{\text {box }}$ and $\tau_{0}$ (curves 1-3 in Figs. 2 2b,c). This is not necessarily in contradiction to the parameter set of Sec. IIF, which was extracted from very central $\mathrm{Pb}+\mathrm{Pb}$ collisions. For the less central NA44 collisions somewat smaller values of $\tau_{0}$ and/or $\Delta \rho$ may indeed be expected.

Given the preliminary nature of the data we did not make a big effort to optimize our fit to the data, e.g. make a combined fit to both proton and deuteron spectra. However, as minimum consistency requirements the lifetime $\tau_{0}$ and the flow velocity $\eta_{f}$ must be large enough to allow for the transverse expansion from the cold $\mathrm{Pb}$ hard sphere radius $(\approx 7 \mathrm{fm})$ to the freeze-out radius $\rho_{\text {box }}$. This is an additional reason to reject parameter choice no. 4.

The outcome of this study is that Gaussian transverse density profiles cannot consistently reproduce both the proton and deuteron spectra, irrespective of the form of the transverse flow profile. A transverse box profile for the density works quite well, both with linear $(\alpha=1)$ and nonlinear ( $\alpha=0.5$ and 2) transverse flow profiles. The source parameters needed to reproduce the deuteron yields and spectra are consistent with those extracted from HBT interferometry with pions.

The physical interpretation of these results is that the data require more nucleons at higher transverse flow than the Gaussian density profile can provide even with steep velocity profiles. Other transverse density profiles like a Woods-Saxon or a doughnut profile may work similarly well or even better; on the other hand, the HBT analysis of pion correlations has so far provided no hint for opaqueness of the source [9]. A good discussion of different profiles, based on the analysis of E802 data from $\mathrm{Si}+\mathrm{Au}$ collisions, can be found in 55.

\section{Au+Au data from the AGS}

Although the size of a cold $\mathrm{Au}$ nucleus is about the same as that of a cold $\mathrm{Pb}$ nucleus, the lower beam energy at the AGS will require different fireball parameters than those given in Sec. IIF, even if only collisions taken at the same $4 \% \sigma_{\text {tot }}$ centrality are considered. A few simple checks with our theoretical results can nevertheless be performed. For E877, Johnson provides for the invariant coalescence factors in the lowest $P_{t}$ bin the values $B_{\mathrm{d}}=$ $(1.5 \pm 0.5) \cdot 10^{-3} \mathrm{GeV}^{2}, B_{3} \mathrm{H}=(1.19 \pm 0.29) \cdot 10^{-6} \mathrm{GeV}^{4}$ and $B_{3} \mathrm{He}=(1.25 \pm 0.23) \cdot 10^{-6} \mathrm{GeV}^{4}$ [32]. $B_{\mathrm{d}}$ is thus about a factor 2 larger than expected for central $\mathrm{Pb}+\mathrm{Pb}$ collisions at the SPS and seen by NA52. Using (6.2) and assuming that the correction factors $\left\langle\mathcal{C}_{A}\right\rangle$ do not differ much between $\mathrm{Pb}+\mathrm{Pb}$ and $\mathrm{Au}+\mathrm{Au}$ collisions, we expect

$$
\left(\frac{B_{2}(\mathrm{Au}+\mathrm{Au})}{B_{2}(\mathrm{~Pb}+\mathrm{Pb})}\right)^{2} \approx\left(\frac{V_{\mathrm{eff}}(\mathrm{Pb}+\mathrm{Pb})}{V_{\mathrm{eff}}(\mathrm{Au}+\mathrm{Au})}\right)^{2} \approx \frac{B_{3}(\mathrm{Au}+\mathrm{Au})}{B_{3}(\mathrm{~Pb}+\mathrm{Pb})} .
$$

These relations work out quite nicely when the $\mathrm{Pb}+\mathrm{Pb}$ estimates (7.1) and the above experimental values for $\mathrm{Au}+\mathrm{Au}$ are inserted. The $\mathrm{E} 877$ value of $B_{2}$ is consistent with the preliminary results $B_{2}=(1.8 \pm 1.0) \cdot 10^{-3} \mathrm{GeV}^{2}$ from E878 for central $\mathrm{Au}+\mathrm{Au}$ collisions at $y=1.7$ and $B_{2}=(0.6 \pm 0.4) \cdot 10^{-3} \mathrm{GeV}^{2}$ from E864 for $10 \% \sigma_{\text {tot }}$ $\mathrm{Au}+\mathrm{Pb}$ collisions at $y=1.968$.

\section{SUMMARY}

We have presented an analytic treatment, supported by numerical checks, of the coalescence of two- and three(anti)nucleon clusters in a relativistically expanding fireball. We showed that coalescence of nuclear clusters and 
two-particle correlations between the final state momenta of pairs of identical particles can be characterized by the same set of effective source parameters, the "lengths of homogeneity". The only additional scale entering the coalescence probability is the intrinsic size of the cluster. We made the connection between coalescence and HBT interferometry explicit with the help of a simple, but phenomenologically successful parametrization of the expanding source which was already extensively studied in connection with two-pion HBT interferometry. For this model most calculations can be done in good approximation analytically.

Starting from the quantum mechanical definition of the deuteron momentum spectrum as a projection of the two-nucleon Wigner density of the fireball at nucleon freeze-out on the deuteron Wigner function, we used the formalism developed by Danielewicz and Schuck [23 to bring the deuteron momentum spectrum into the form (3.19) of a modified Cooper-Frye formula. Except for the "quantum mechanical correction factor" $\mathcal{C}\left(R_{\mathrm{d}}, P_{\mathrm{d}}\right)$ this formula looks exactly like a thermal spectrum of elementary particles with the mass of the deuteron, where the deuteron phase-space distribution is given by a product of thermal neutron and proton phase-space distributions. The factor $\mathcal{C}_{\mathrm{d}}$ is given by the integral 3.20 of the deuteron Wigner density over the homogeneity volume of the nucleon source; for each deuteron momentum $P_{\mathrm{d}}$ it is peaked at the value $R_{\mathrm{d}}$ at which the flow velocity equals the deuteron velocity. We showed that the peak value for deuterons at rest in the fireball frame is a good approximation for $\left\langle\mathcal{C}_{\mathrm{d}}\right\rangle$, the average of $\mathcal{C}_{\mathrm{d}}$ over the freeze-out hypersurface, which is the relevant quantity for the shape of the deuteron momentum spectrum. $\left\langle\mathcal{C}_{\mathrm{d}}\right\rangle$ can be expressed by the very simple formula (4.12) in terms of the ratios between the deuteron size $d$ and the longitudinal and transverse lengths of homogeneity for nucleons at rest, $\mathcal{R}_{\|}(m)$ and $\mathcal{R}_{\perp}(m)$, respectively. In our expanding fireball model $\langle\mathcal{C}\rangle$ turns out to be essentially momentum-independent for clusters of all sizes, and is of order $\langle\mathcal{C}\rangle \sim 0.7-0.8$ for 2- and 3-nucleon clusters formed in $160 A \mathrm{GeV} \mathrm{Pb}+\mathrm{Pb}$ collisions.

The shape of the deuteron momentum spectrum is thus given by the thermal model ansatz $(2.3)$, with $\langle\mathcal{C}\rangle$ leading only to a modification of the normalization. For our Gaussian source parametrization the single particle spectrum can be written, up to trivial factors, as a product (2.14b) of an exponential in $M_{t}$ with inverse slope ("effective temparature" ) $T^{*}$ (2.16), a Gaussian in rapidity with width $(\Delta \eta)^{2} / A$, and an effective volume $V_{\text {eff }}\left(A, M_{t}\right)$ which is again given by the homogeneity lengths [47, see (2.19). This allows to express the invariant coalescence factor $B_{2}$ fully in terms of the deuteron size and the lengths of homogeneity, see (6.3).

Unfortunately, the Gaussian model (Gaussian transverse density profile with linear transverse flow rapidity profile) leads to identical $M_{t}$-slopes for clusters of all sizes and therefore to $B_{A}$ values which are essentially momentum-independent. This is contradicted by exper- iment. We found, in agreement with Polleri et al. [55], that the flatter deuteron than proton spectra require a density profile which gives for larger clusters more weight to regions of larger transverse flow. We found that a transverse box profile works very well and successfully reproduces the measured slope of preliminary data [0, 8 for the deuteron $M_{t}$-spectrum in $\mathrm{Pb}+\mathrm{Pb}$ collisions. The consequence is a momentum-dependence of $B_{2}$ which now rises as a function of $M_{t}$ according to the simple generalized expression (6.4).

It is interesting to observe that in this way the cluster spectra provide additional information about the source which cannot be extracted from HBT measurements. The latter constrain only the rms radii of the effective source, but not the shape of its spatial distribution. In the meantime, however, it has been found that a combined analysis of pion spectra and HBT correlations also prefers a transverse box profile over a Gaussian one because the former gives a larger total pion yield than the latter, as required by the data [71].

\section{ACKNOWLEDGMENTS}

The authors want to thank S. Kabana, R. Klingenberg, and M. Murray for discussing with us their preliminary data prior to publication. This work was supported by GSI, BMBF and DFG.

[1] S. Mrówczyński, Phys. Lett. B 248, 459 (1990); 277, 43 (1992); and 345, 393 (1995).

[2] A.N. Makhlin and Yu.M. Sinyukov, Z. Phys. C 39, 69 (1988); S.V. Akkelin and Yu.M. Sinyukov, Phys. Lett. B 356, 525 (1995).

[3] For recent reviews and references to the extensive literature describing the application of this method to heavy ion collisions see U. Heinz, Nucl. Phys. A 610, 264c (1996); and U. Heinz, in CRIS'98: Measuring the size of things in the universe: HBT interferometry and heavy ion physics, eds. S. Costa et al. (World Scientific, Singapore, 1998, in press), hep-ph/9806512.

[4] S. Kabana et al., Newmass (NA52) Collaboration, Nucl. Phys. A 638, 411c (1998).

[5] Proceedings of "Quark Matter '97", (Tsukuba, Japan, Dec. 1-5, 1997), eds. T. Hatsuda et al., Nucl. Phys. A 638 (1998)

[6] S. Kabana, NA52 Collaboration (private communication).

[7] M. Murray et al., NA44 Collaboration, in: 3rd International Conference on Physics and Astrophysics of Quark-Gluon Plasma, 17-21 March, 1997, Jaipur, India, nucl-ex/9706007.

[8] M. Murray, NA44 Collaboration (private communication). 
[9] U.A. Wiedemann, B. Tomášik, and U. Heinz, Nucl. Phys. A 638, 475c (1998).

[10] H. Appelshäuser et al. (NA49 Collaboration), Eur. J. Phys. C 2, 661 (1998).

[11] S.T. Butler and C.A. Pearson, Phys. Rev. 129, 836 (1963).

[12] A. Schwarzschild and Č. Zupančič, Phys. Rev. 129, 854 (1963).

[13] H.H. Gutbrod et al., Phys. Rev. Lett. 37, 667 (1976).

[14] R. Bond, P.J. Johansen, S.E. Koonin and S. Garpman, Phys. Lett. B 71, 43 (1977).

[15] A.Z. Mekjian, Phys. Rev. Lett. 38, 640 (1977); Phys. Rev. C 17, 1051 (1978); and Nucl. Phys. A 312, 491 (1978).

[16] J.I. Kapusta, Phys. Rev. C 21, 1031 (1980).

[17] H. Sato and K. Yazaki, Phys. Lett. B 98, 153 (1981).

[18] E. Remler, Ann. Phys. 136, 293 (1981); M. Gyulassy, K. Frankel, and E.A. Remler, Nucl. Phys. A 402, 596 (1983).

[19] L.P. Csernai and J.I. Kapusta, Phys. Rep. 131, 223 (1986).

[20] S. Mrówczyński, J. Phys. G 13, 1089 (1987); and Phys. Lett. B 308, 216 (1993).

[21] C.B. Dover, U. Heinz, E. Schnedermann, and J. Zimányi, Phys. Rev. C 44, 1636 (1991) 1636

[22] P. Danielewicz and G.F. Bertsch, Nucl. Phys. A 533, 712 (1991).

[23] P. Danielewicz and P. Schuck, Phys. Lett. B 274, 268 (1992).

[24] S. Leupold and U. Heinz, Phys. Rev. C 50, 1110 (1994).

[25] W.J. Llope et al., Phys. Rev. C 52, 2004 (1995).

[26] J.L. Nagle et al., Phys. Rev. C 53, 367 (1996).

[27] R. Klingenberg et al., Newmass (NA52) Collaboration, Nucl. Phys. A 610, 306c (1996).

[28] D.E. Kahana et al., Phys. Rev. C 54, 338 (1996).

[29] R. Mattiello et al., Phys. Rev. C 55, 1443 (1997).

[30] H. Sorge, J.L. Nagle, and B.S. Kumar: Phys. Lett. B 355, 27 (1995).

[31] F. Cooper and G. Frye, Phys. Rev. D 10, 186 (1974).

[32] S.C. Johnson, E877 Collaboration, PhD thesis, SUNY Stony Brook, October 1997; S.C. Johnson et al. (E877 Collaboration), in 33.

[33] Proceedings of the $3^{\text {rd }}$ HIPAGS Workshop (Heavy Ion Physics at the AGS), Aug. 22-24, 1996, Wayne State University, Detroit, Michigan (http://rhic.physics.wayne.edu/hipags96/).

[34] M.L. Goldberger and K.M. Watson, Collision Theory (Wiley, New York, 1964).

[35] R. Dashen, S. Ma, and H.J. Bernstein, Phys. Rev. 187, 345 (1969).

[36] M. Schmidt, G. Röpke, and H. Schulz, Ann. Phys. (N.Y.) 202, 57 (1990).

[37] R. Hagedorn, Phys. Rev. Lett. 5, 276 (1960); Nuovo Cimento 25, 1017 (1962); and Suppl. Nuovo Cimento 3, 147 (1965).

[38] P. Sonderegger, R. Shahoian, S. Albergo, in CRIS'96: Critical Phenomena and Collective Observables, eds. S. Costa et al., p. 225 (World Scientific, Singapore, 1996).

[39] K.S. Lee, U. Heinz, and E. Schnedermann, Z. Phys. C 48, 525 (1990); E. Schnedermann, J. Sollfrank, and U. Heinz,
Phys. Rev. C 48, 2462 (1993).

[40] L.V. Bravina et al., Phys. Lett. B 434379 (1998); L.V. Bravina et al., J. Phys. G 25, 351 (1999).

[41] E. Schnedermann and U. Heinz, Phys. Rev. C 50, 1675 (1994).

[42] U. Mayer and U. Heinz, Phys. Rev. C 56, 439 (1997).

[43] J.D. Bjorken, Phys. Rev. D 27, 140 (1983).

[44] B. Tomášik and U. Heinz, nucl-th/9805016.

[45] Rüdiger Scheibl, PhD thesis, Universität Regensburg, 1998, available from http://www.physik.uni-regensburg.de/ ${ }^{\sim}$ scr04317/

[46] S. Chapman, P. Scotto, and U. Heinz, Heavy Ion Physics 1, 1 (1995).

[47] T. Csörgő and B. Lörstad, Phys. Rev. C 54, 1396 (1996).

[48] U.A. Wiedemann, P. Scotto, and U. Heinz, Phys. Rev. C 53, 918 (1996).

[49] J.R. Nix, Phys. Rev. C 58, 2303 (1998).

[50] $\mathrm{Nu} \mathrm{Xu}$ for the NA44 Collaboration, Nucl. Phys. A 610, $175 c$ (1996).

[51] I.G. Bearden et al., NA44 Collaboration, Phys. Rev. Lett. 78, 2080 (1997).

[52] P. Vincent et al., E802 Collaboration, Nucl. Phys. A 498, 67c (1989); T. Abbott et al., E802 Collaboration, Phys. Rev. C 50, 1024 (1994).

[53] J. Simon-Gillo et al., NA44 Collaboration, Nucl. Phys. A 590, 483c (1995).

[54] G. Roland et al., NA49 Collaboration, Nucl. Phys. A 638, 91c (1998).

[55] A. Polleri, J.P. Bondorf, and I.N. Mishustin, Phys. Lett. B 419, 19 (1998).

[56] Landolt-Börnstein: Gruppe I (Kernphysik und Kerntechnik), Band 2 (Kernradien) (Springer Verlag, Berlin, 1967); P. Ring and P. Schuck, The nuclear many-body problem (Springer Verlag, New York, 1980).

[57] S. Chapman, J.R. Nix, and U. Heinz, Phys. Rev. C 52, 2694 (1995); U. Heinz, B. Tomášik, U.A. Wiedemann, and Y.-F. Wu, Phys. Lett. B 382, 181 (1996); Y.-F. Wu, U. Heinz, B. Tomášik, and U.A. Wiedemann, Eur. Phys. J. C 1, 599 (1998).

[58] S. Schönfelder, PhD thesis, MPI für Physik, München, 1996, available from the NA49 home page, (http://na49info.cern.ch), NA49 note number 143 (15.01.98).

[59] H. Appelshäuser, PhD thesis, J.W. Goethe-Universität, Frankfurt am Main, 1996, available from the NA49 home page (http://na49info.cern.ch), NA49 note number 150 (10.02.98).

[60] T. Ericsson and W. Weise, Pions and Nuclei (Clarendon Press, Oxford, 1988).

[61] R.P. Feynman, Statistical mechanics: a set of lectures (Benjamin, MA, 1972).

[62] E. Wigner, Phys. Rev. 40, 749 (1932).

[63] E.A. Remler, in The physics of phase space, eds. Y.S. Kim and W.W. Zachary (Springer, Berlin, 1986).

[64] B. Lörstad, Int. J. Mod. Phys. A 12, 2861 (1989).

[65] A.T.M. Aerts and C.B. Dover, Phys. Rev. D 28, 450 (1983).

[66] B. Kämpfer, hep-ph/9612336.

[67] G. Appelquist et al., Newmass (NA52) Collaboration, Phys. Lett. B 417, 202 (1998). 
[68] J.K. Pope at al., E864 Collaboration, in Ref. [33].

[69] S. Kabana for the NA52 collaboration, J. Phys. G 25, 217 (1999).

[70] A. Sakaguchi et al., NA44 Collaboration, Nucl. Phys. A 638, 103c (1998).

[71] B. Tomášik, PhD thesis, Universität Regensburg, 1999; and B. Tomášik and U. Heinz, to be published.

TABLE I. The quantum mechanical correction factor $\mathcal{C}_{\mathrm{d}}^{0}$ for Hulthen and harmonic oscillator wave functions calculated with Eq. (3.21), for different fireball parameters at nucleon freeze-out (for details see text).

\begin{tabular}{l|ccc|ccc}
\hline \hline$\tau_{0}[\mathrm{fm} / c]$ & & 9.0 & & & 6.0 & \\
\hline$T[\mathrm{MeV}]$ & 168 & 130 & 100 & 168 & 130 & 100 \\
$\eta_{f}$ & 0.28 & 0.35 & 0.43 & 0.28 & 0.35 & 0.43 \\
\hline Hulthen & 0.86 & 0.84 & 0.80 & 0.80 & 0.78 & 0.74 \\
harm. osc. & 0.84 & 0.81 & 0.76 & 0.76 & 0.72 & 0.66 \\
\hline \hline
\end{tabular}



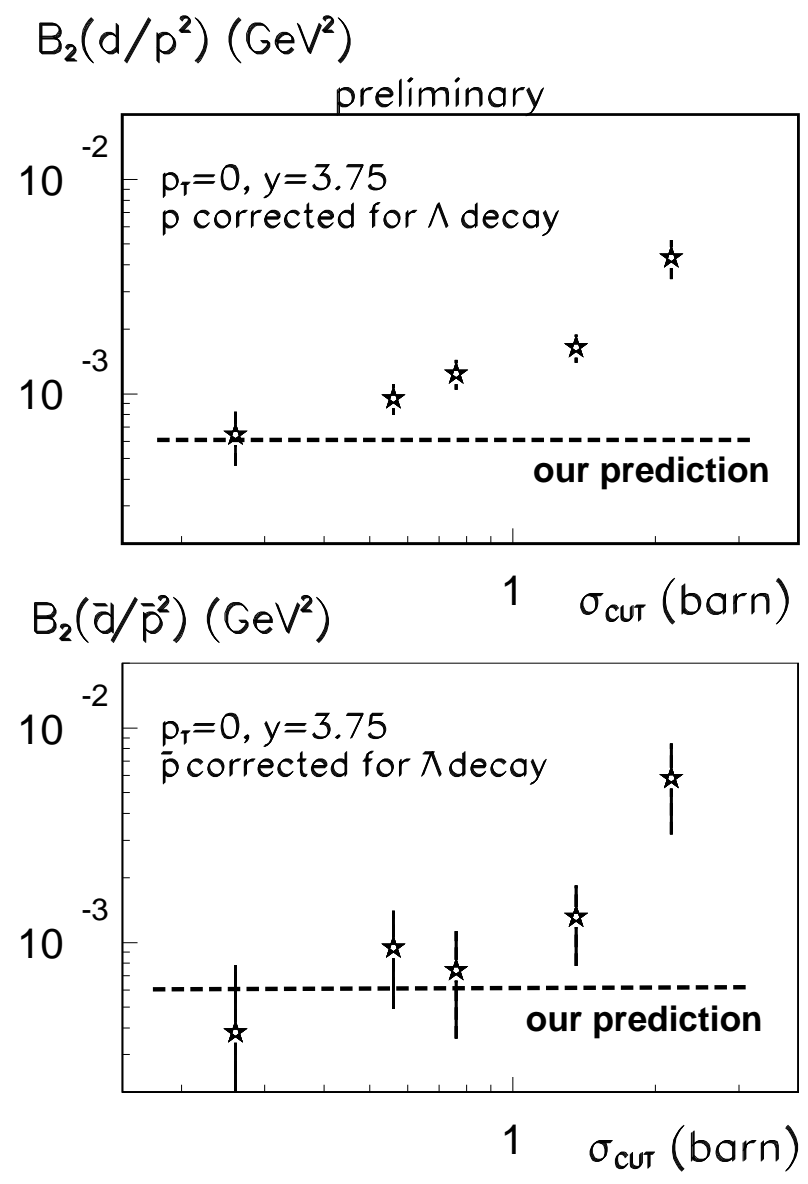

FIG. 1. $B_{2}$ for deuterons and antideuterons in $158 \mathrm{~A} \mathrm{GeV} \mathrm{Pb}+\mathrm{Pb}$ collisions as a function of the centrality, measured by NA52 [4]. (The vertical scale has a systematic error of $\sim 40 \%$.) With $\sigma_{\text {tot }}^{\text {inel }} \approx 8.2$ barn in Pb+Pb, the left-most data points correspond to a centrality of about $4 \% \sigma_{\text {tot }}$. The dashed lines show our prediction for $B_{2}$ at $4 \% \sigma_{\text {tot }}$ (see text). 

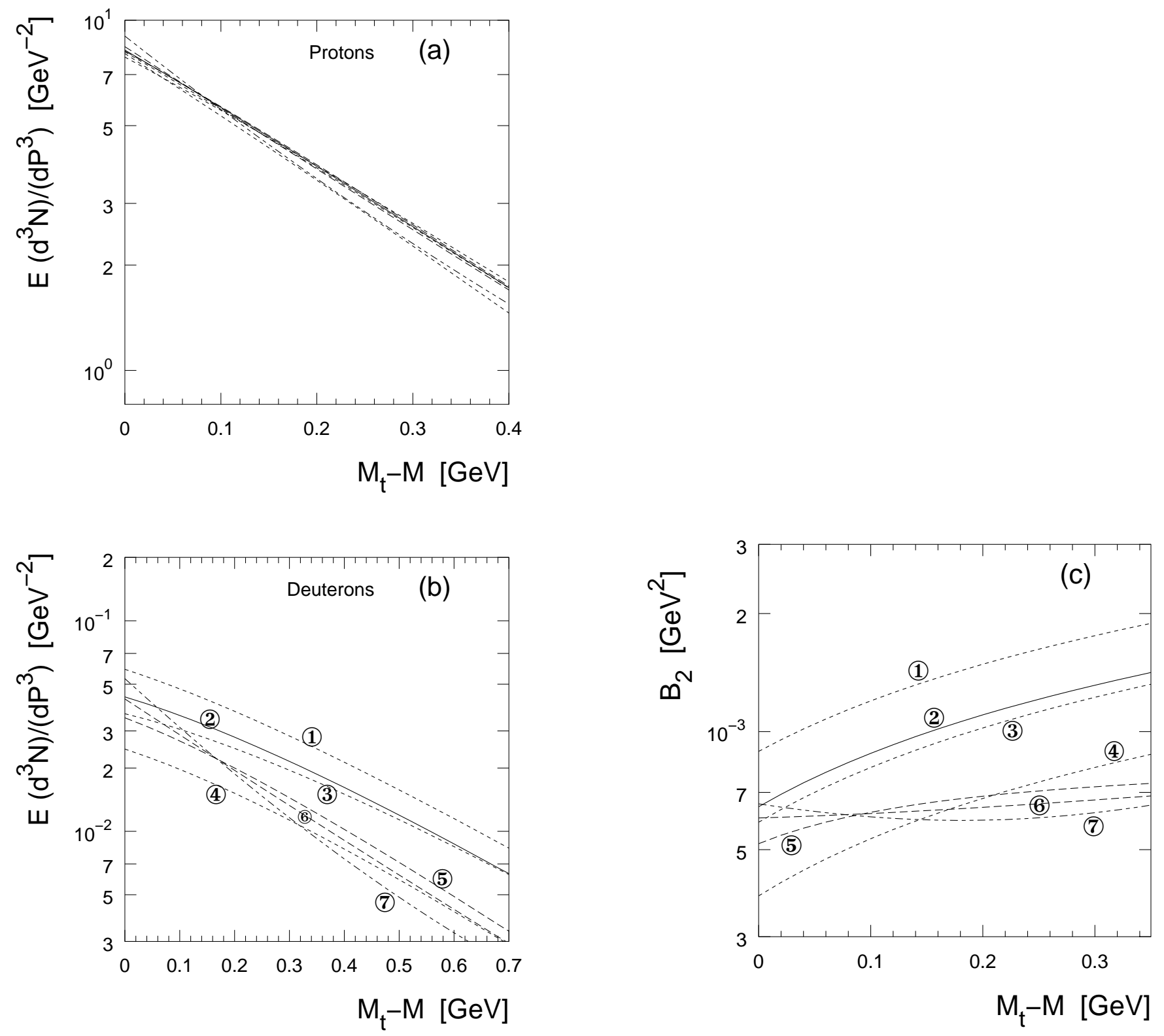

FIG. 2. Fits to the proton $m_{t}$-spectrum (a) and predicted deuteron $M_{t}$-spectra (b) and the ratio $B_{2}=\mathrm{d} / \mathrm{p}^{2}$ (c) for different models for the transverse density profile. The analysis was performed with preliminary data (not shown) from the NA44 Collaboration 8] for $20 \% \sigma_{\text {tot }} \mathrm{Pb}+\mathrm{Pb}$ collisions at $158 \mathrm{AGeV}$ in the rapidity range $1.9 \leq y \leq 2.3$. Lines $1-4$ : box profile for the transverse density with linear transverse flow profile; lines 5-7: Gaussian transverse density profile with power law transverse flow profiles, with exponents $\alpha=0.5, \alpha=1.0$ and $\alpha=2.0$. The preliminary data lie close to line 2. For details see text. 